



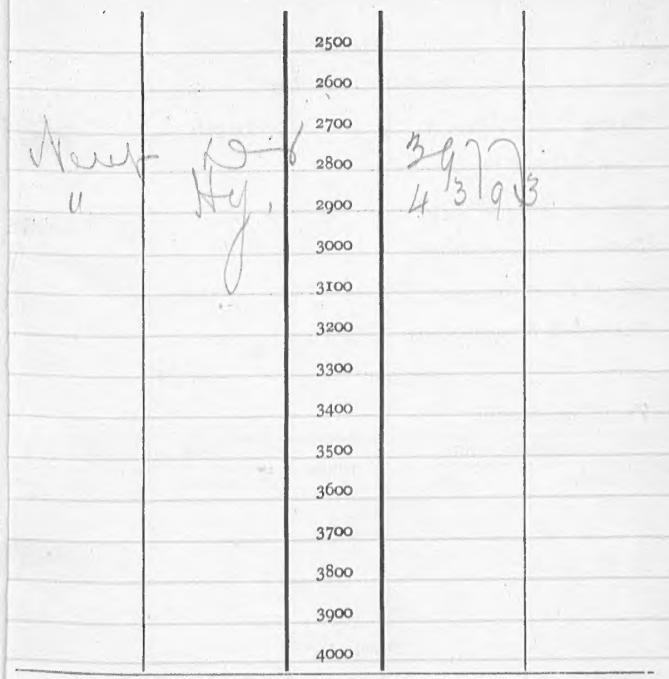

SERIAL TEMPERATURES:

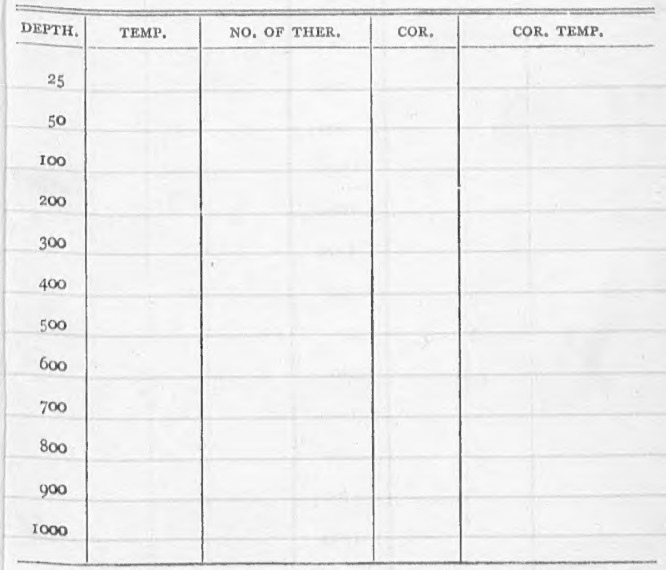

REMARKS : 


\section{enot 011}

No. 1393 aifNo $\bigcup$ Machine. I Reel. Turns $2 / 3$ cor $+2 \frac{2}{41}$ shot on zead 60 A Bottiom co.co. 8. SN.

Bottom temperature

No. of thermometer 50460 Cor.

\section{Corrected temperature}

Air 80 Storface To Drift

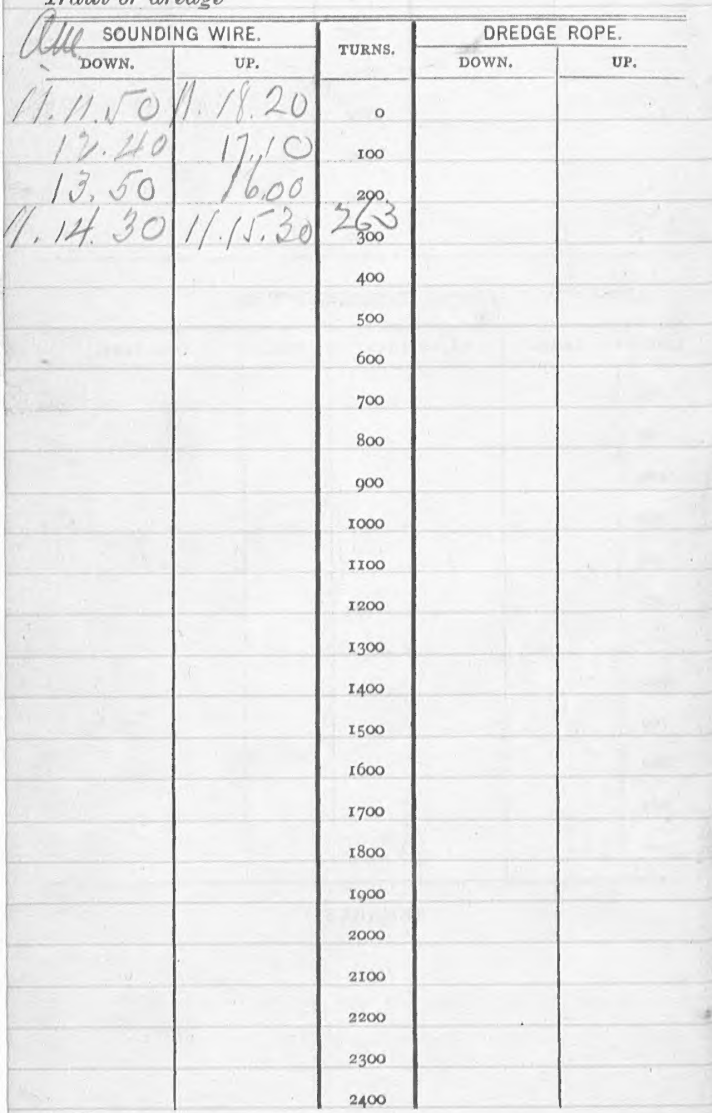


Mordu Mamu or Bria Sland-7icinity of

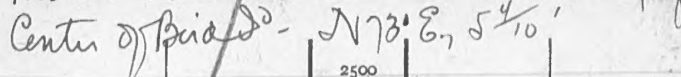

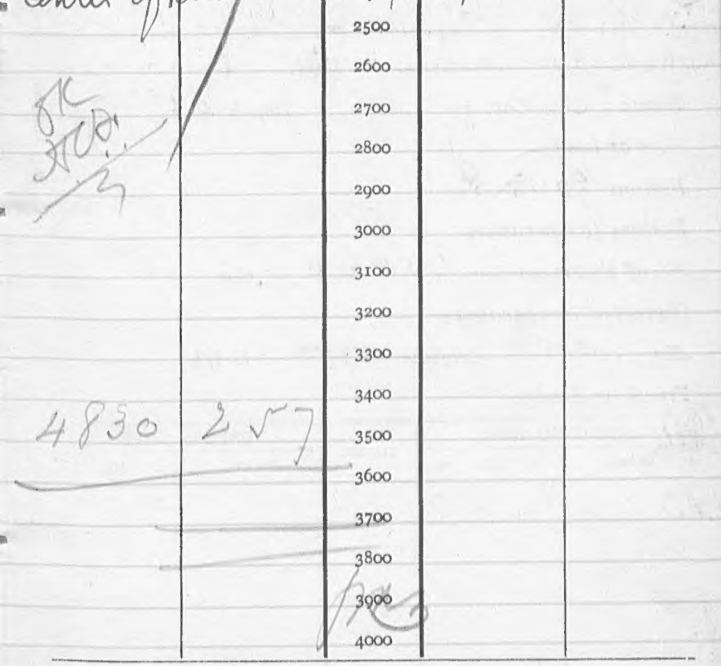

SERIAL TEMPERATURES,

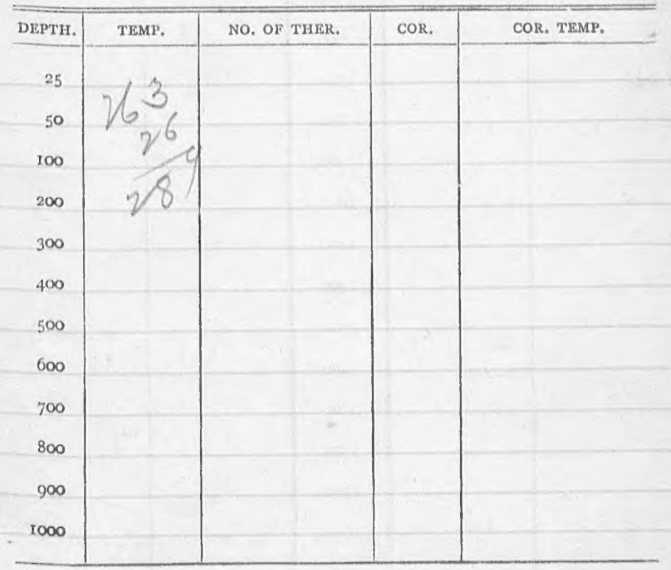

REMARKS : 


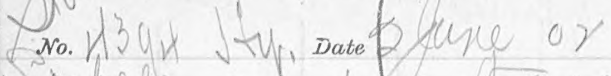
Iypold Machine. I keel. $\sqrt{ }$ a a

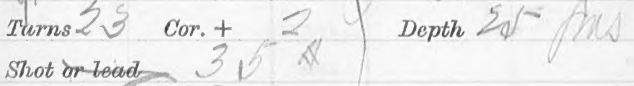

Bottom $6 a, 8$,

Bottom temperature

No. of thermometer.

Cor.

Corrected temperature

Air 80 surface $7 \sqrt{ }$ Drift

Trawl or dredge

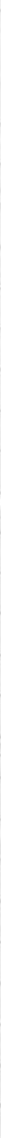





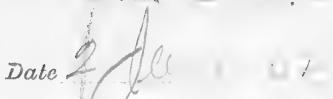

Machine.

Tiecl.

Turns 23 Cor. + Depth

S7ubrar lead

Bottom

Bottom temperature

No. of thermometer

Cor.

Corrected temporature

Air $\$ 0$ Sierface

Drift

Trawl or dredge

SOUNDING WIRE.

DOWN.

UT.

$\sqrt{2}+2,8$

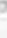

\section{;} V 
No. Date

1Pッn! Machine. - Reel.

Turns 2/ Cor + /

Depth 22

stutor lead is 6 C

Bottom $\quad Q_{0}=\omega_{0}$.

Bottom temperature

No. of thermometer

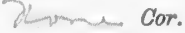

Corrected temperature

Air 80 ... Surface . D Drift

Trawl or areage

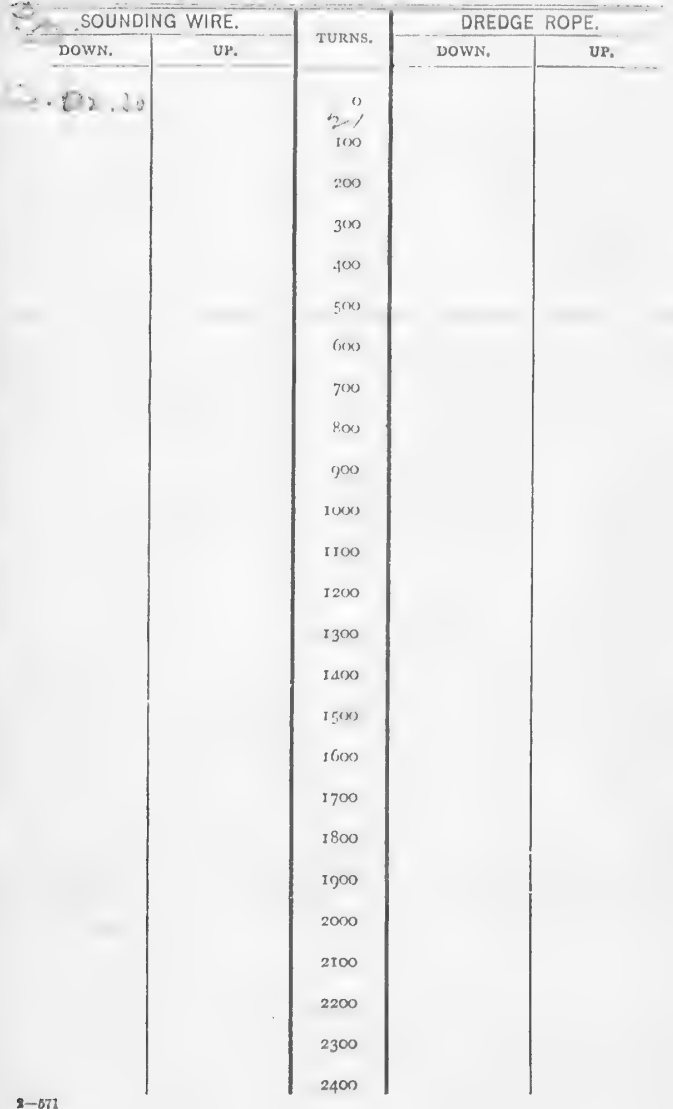




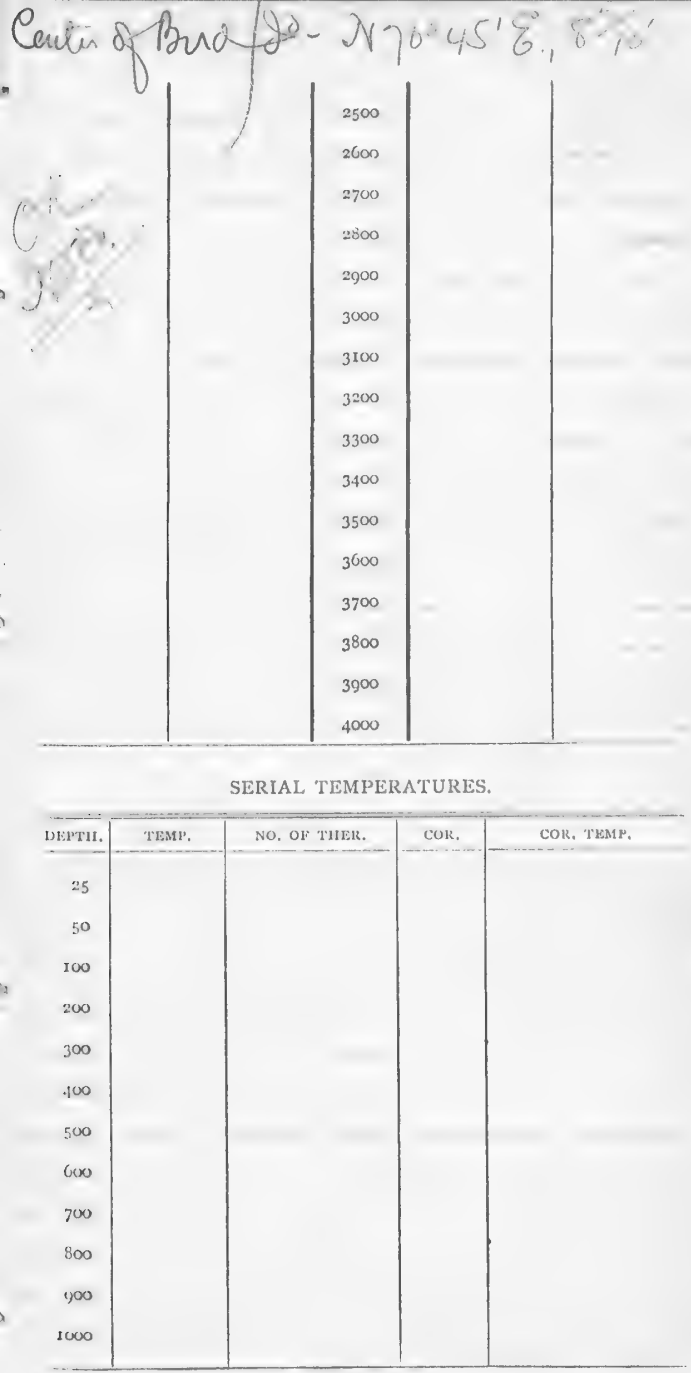

REMARKS: 
( $\therefore \because 4$, Machine. - Reel. -

Turns 21 Cor. + 1 Depth 2 \& fiv

andead ofor.

Bottom S. Q.

Bottom temperature

No. of thermometer

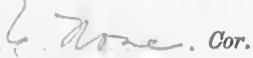

Corrected temperatuke
Air
Surface
Drift

Trawl or dredge

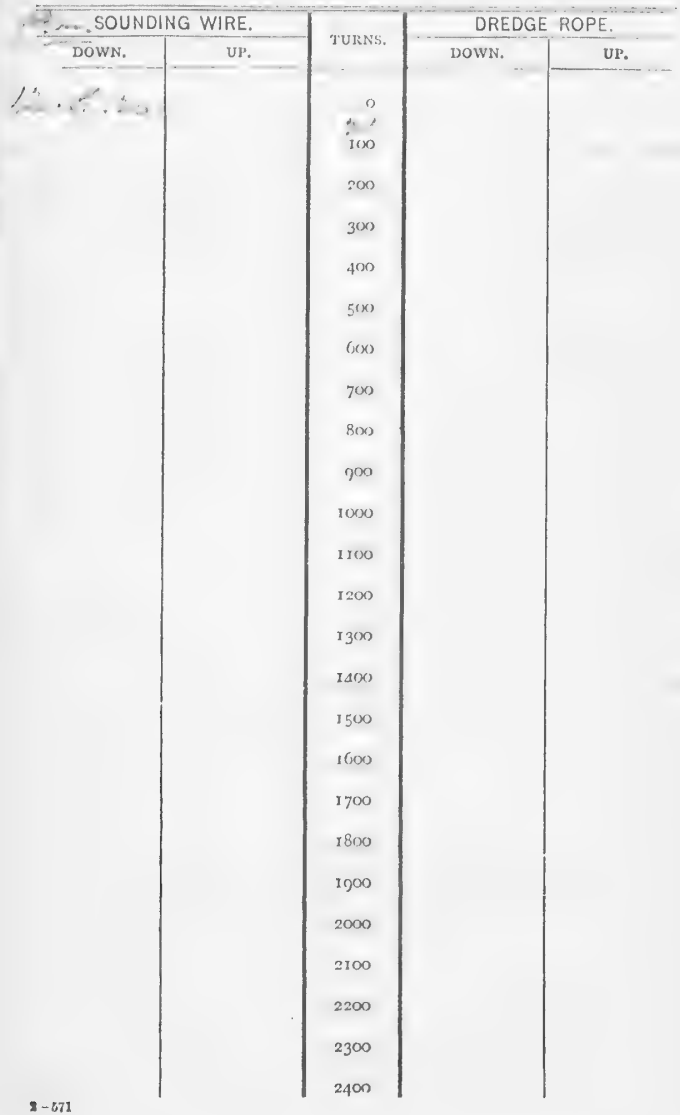




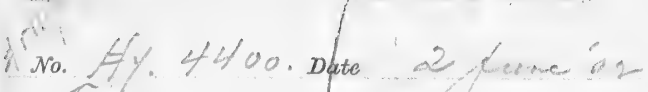
Avier Machine - Reel
Turns 2/ Cor. +

Shot or lead 26 Con

Bottiom

Co 5 .

Bottom temperature

No. of thernometer

Munc cor.

Corrected temperature

Air 80 Surface $7 \sqrt{\text { Drift }}$

Trawt or dredge

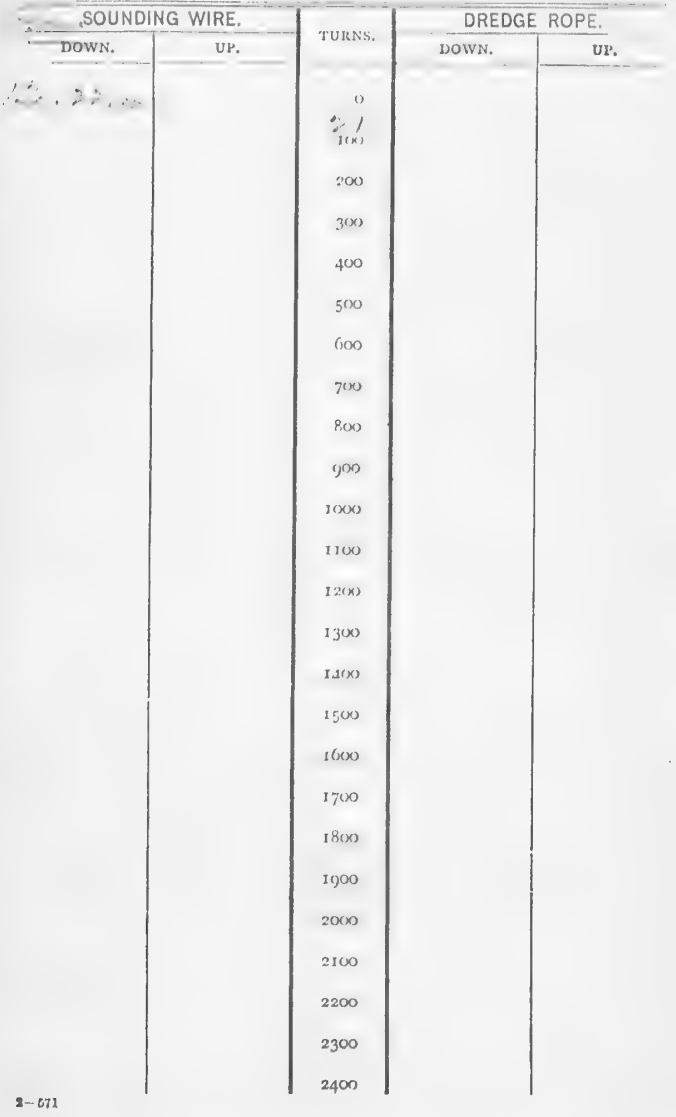




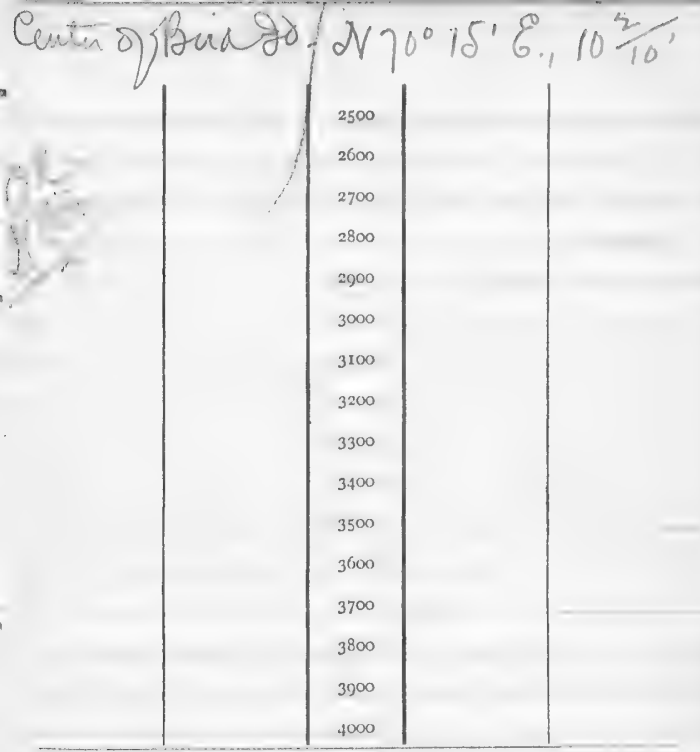

SERIAL TEMPERATURES.

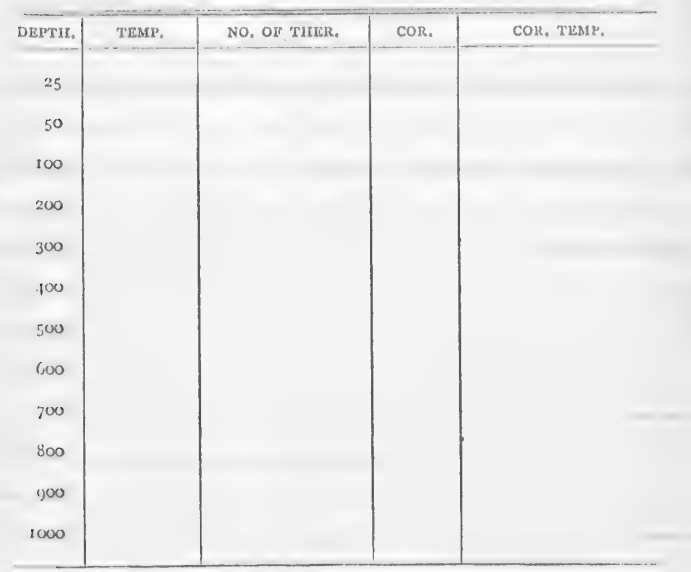

REMARKS : 
No.

\section{Date}

Tanner Machine -Reel.

Turns Cor. +

Shot or lead

26

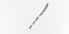

Depth

Bottom $\mathrm{C}$.

Bottom temperature

No. of thermometer

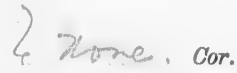

Corrected temperature

Air..8O Surface

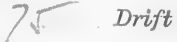

Trawl or dredgo

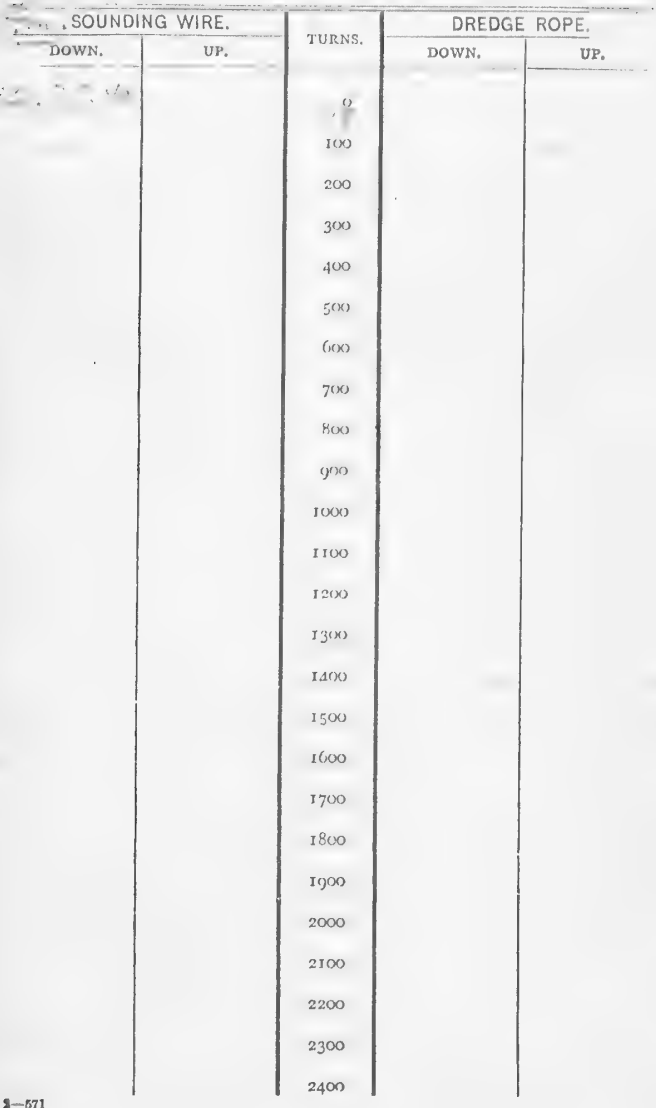


No. 2 . I 402 Date 2 : Nétse, Machine, Reel.

Turns Cor. + Depth

stot lead $26 \quad C_{b}$.

Bottom C.

Bottom temperature? No. of thermometer

Cor.

Corrected temperaturies

Air 80 Surface 75 Drift

Trawl or dredge

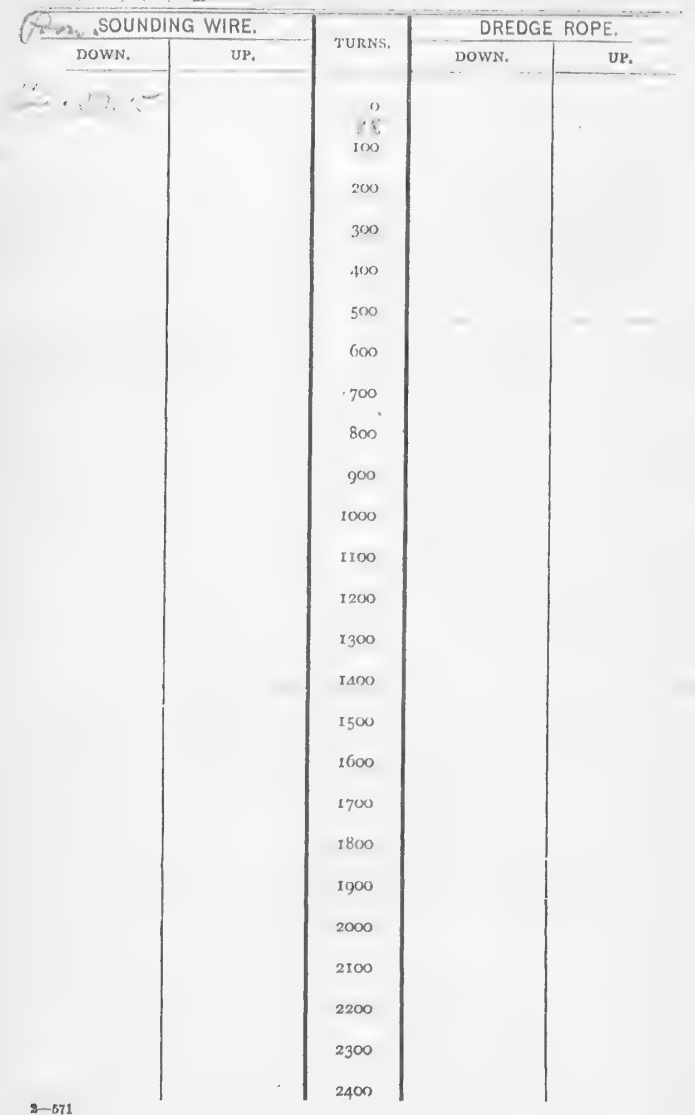




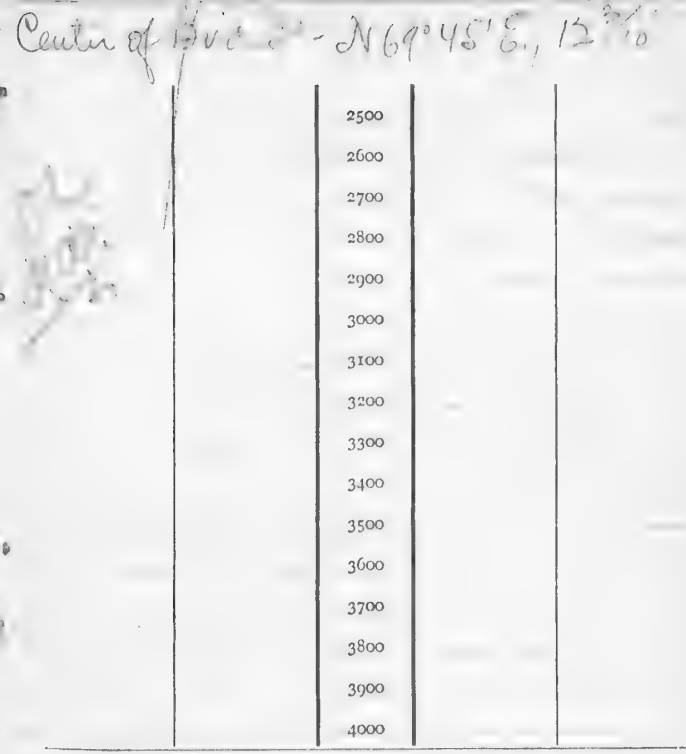

SERIAL TEMPERATURES.

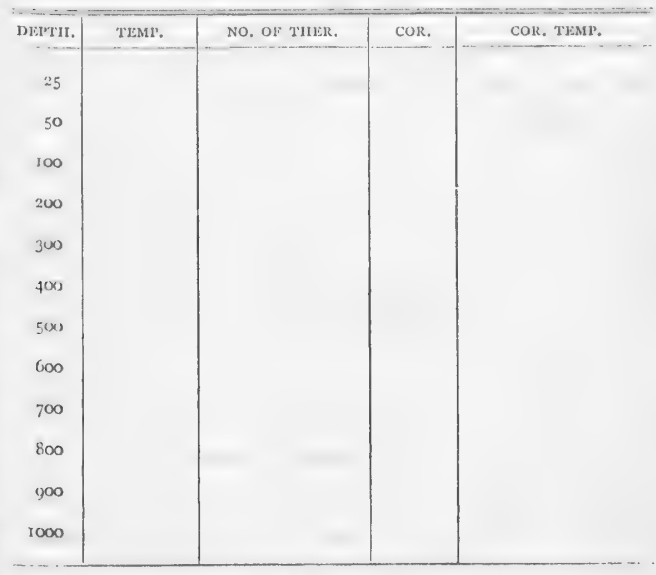

REMARKS : 
No.

\section{Dule}

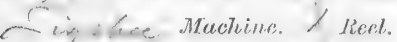

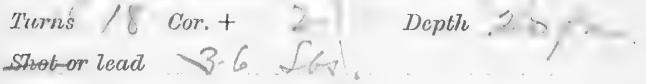

Bottiom

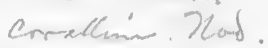

Brottom temperature

No. of thermometer

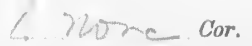

Corrected temperature.

Air 77. Surface $7 \sqrt{\text { Drift }}$

Trawt or dredge

$\therefore$ SOUNDING WIRE.

DOWN. UP.

UP.

TURNS,

DREDGE ROPE.

, , .....

-

is

Ion

200

300

400

500

600

700

800

900

1000

y100

1200

1300

1400

I500

1600

1700

1800

1000

2000

$21(x)$

$22(x)$

2300

2400

UP. 


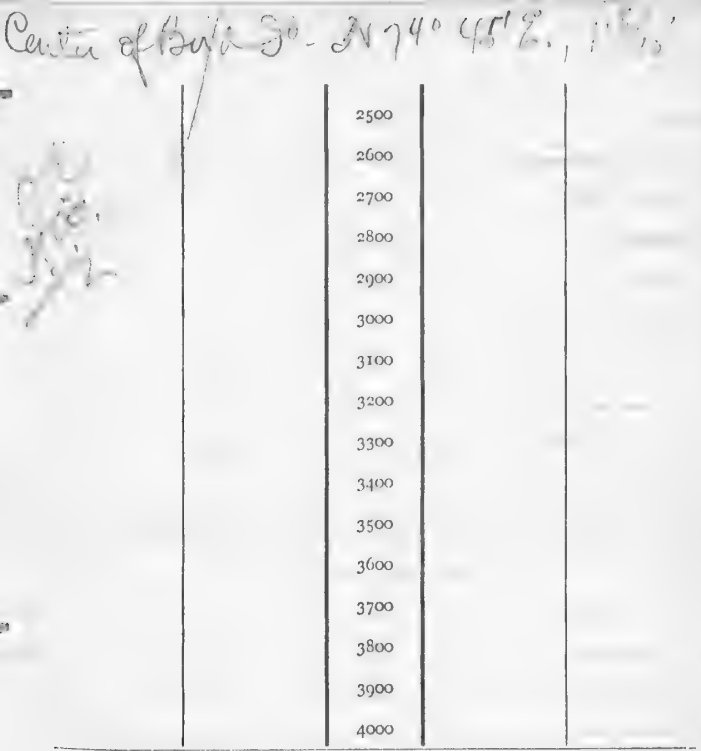

SERIAL TEMPERATURES.

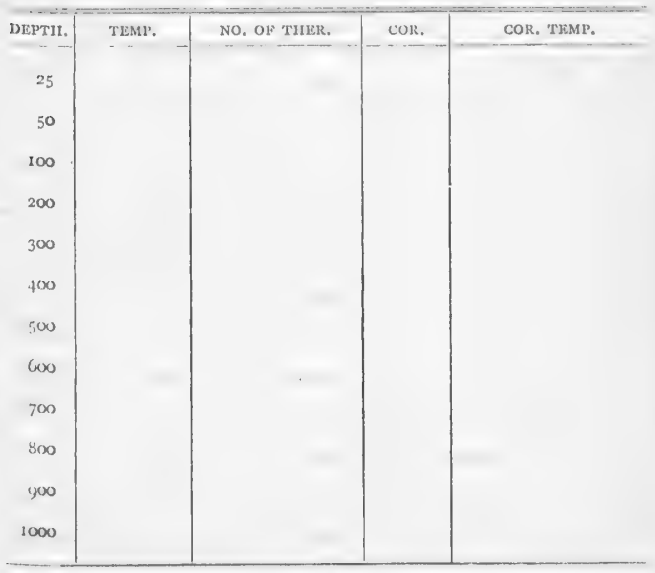

REMARKS : 
nor.

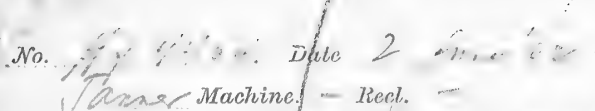

Rect
Therns
Cor. +
Deplh

S7loter lead

Bottom

2.

Bottom temperature

No. of thormometer

Herme Cor.

Corrected temperature

Air T\%. Surface 7.- - Drift

Trawl or dredge

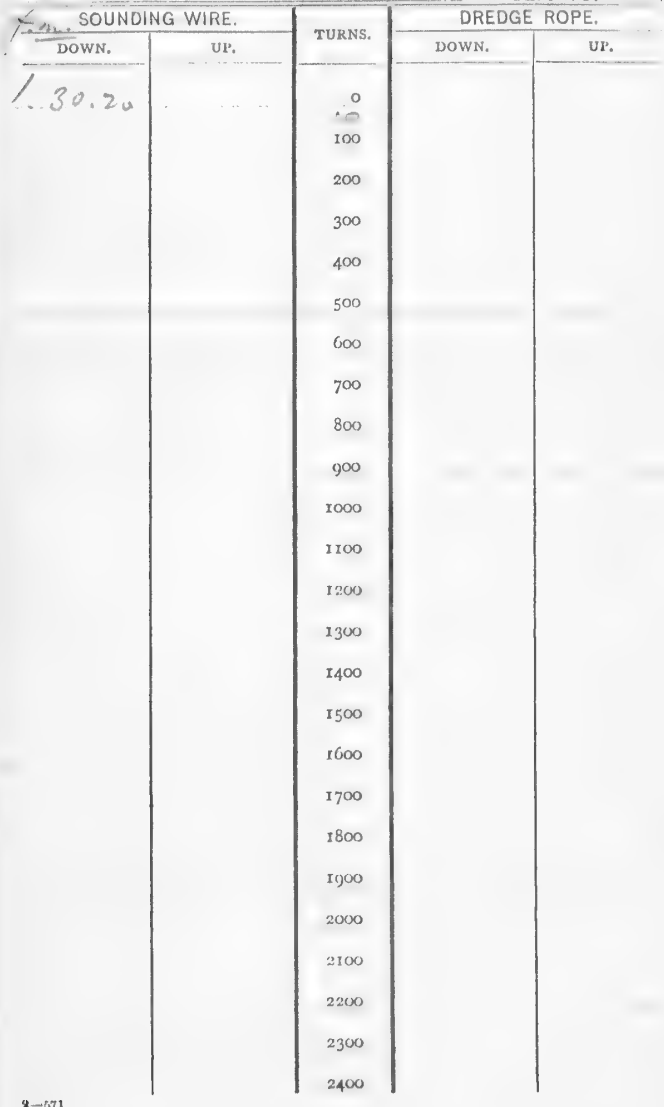


No.

$$
\text { \%०० Date }
$$

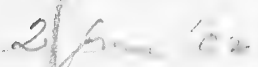

|VMave Machine. - Reel. -
Turus $=0$ Cor. +
2 Depth

sleatur lead 26

Botitom

ers cos.

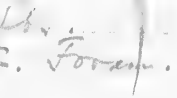

Bottom temperature

No. of thermometer

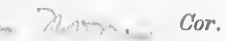

Corrected temperature

Air . J\% - Strrface . 75. Drift

Trawl or dredge

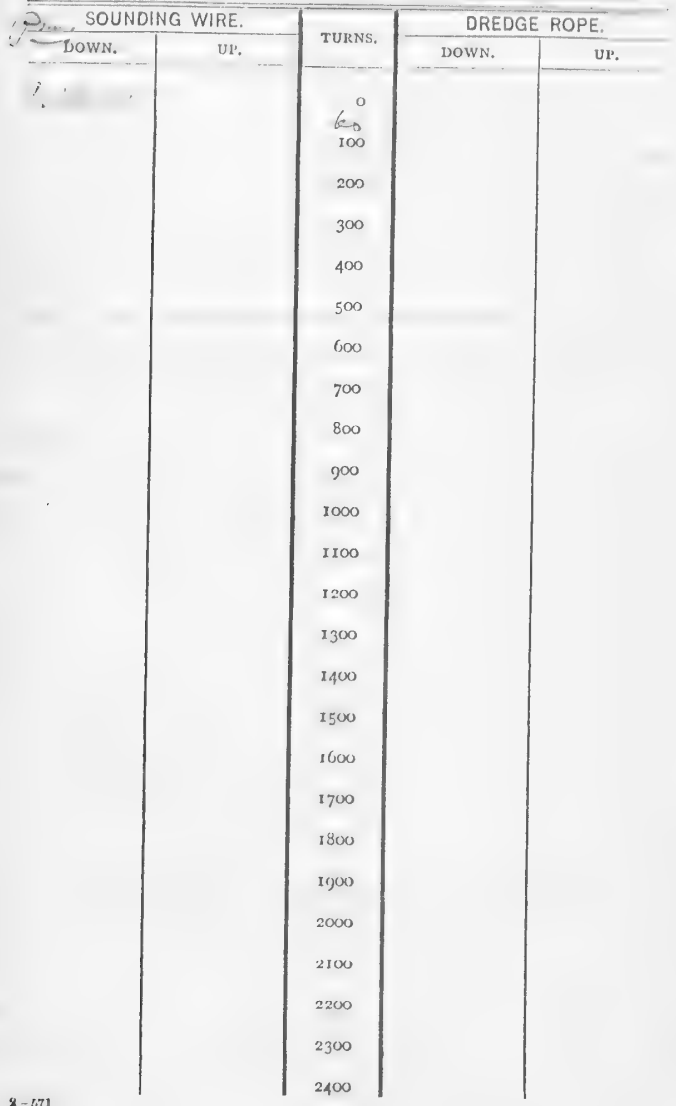


No.

Dite

"Xiqué Machine." / Reel. Var.

Turns 333 Cor +33 Depth 366 Fw.

कीow lead $36 . \sqrt{6}$.

Bottom fre co $S$.

Bottom temperature

No.of thermometer $Y$ Nonc Cor.

Corrected temperature

Air 78. - Storface 75. Drift

Trawl or dredge

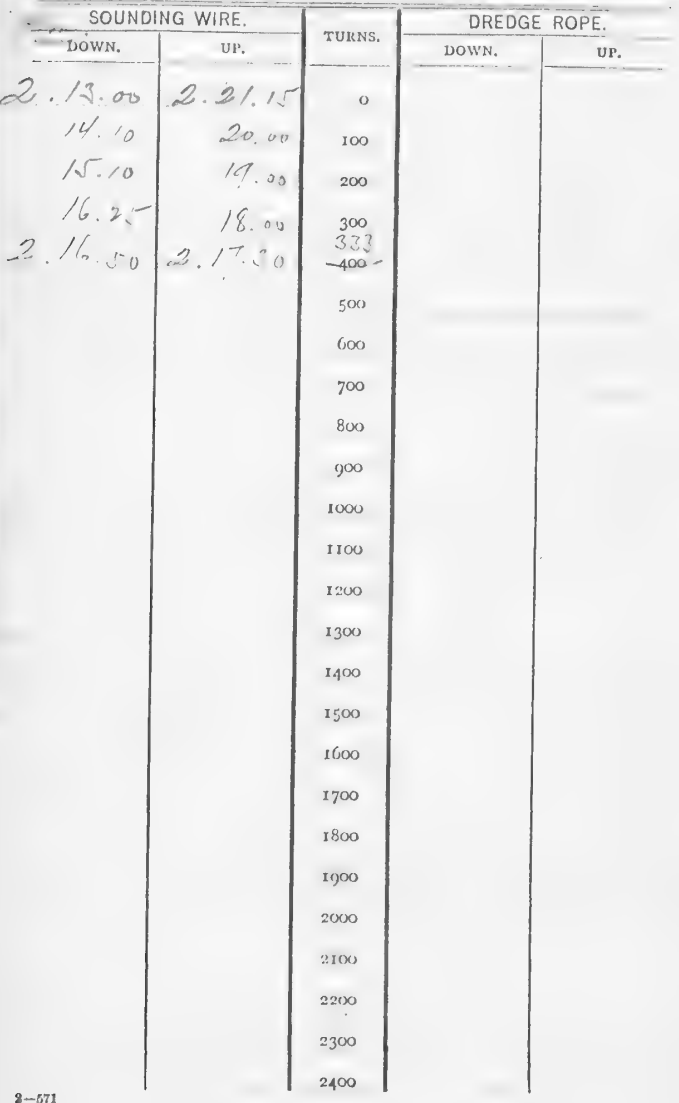


Centa of Bund $\& 85^{\circ} \varepsilon, 11^{\prime \prime} 10$

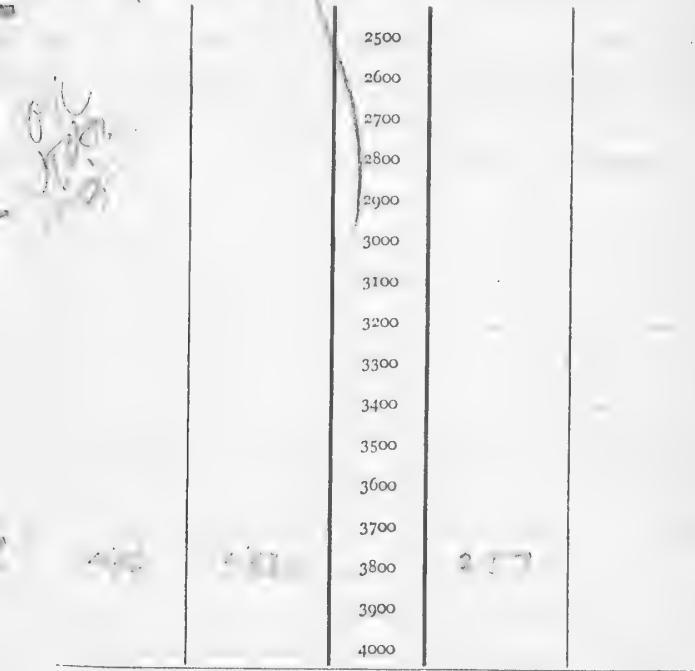

SERIAL TEMPERATURES.

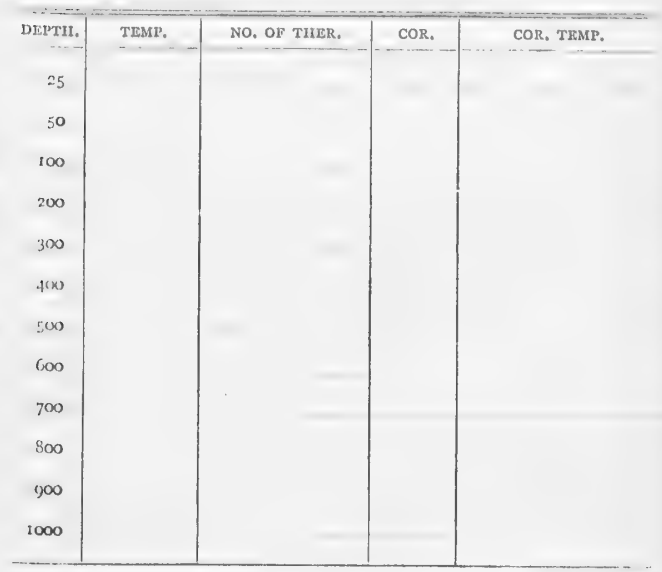

REMARKS : 


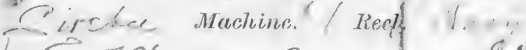

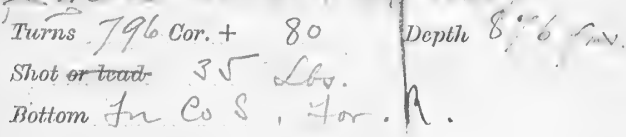

Bottom temperature

No. of thormometer. 80460

Cor.

Corrected temperature

Air . 78. Surface J . Drift - I!

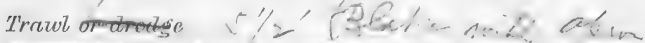

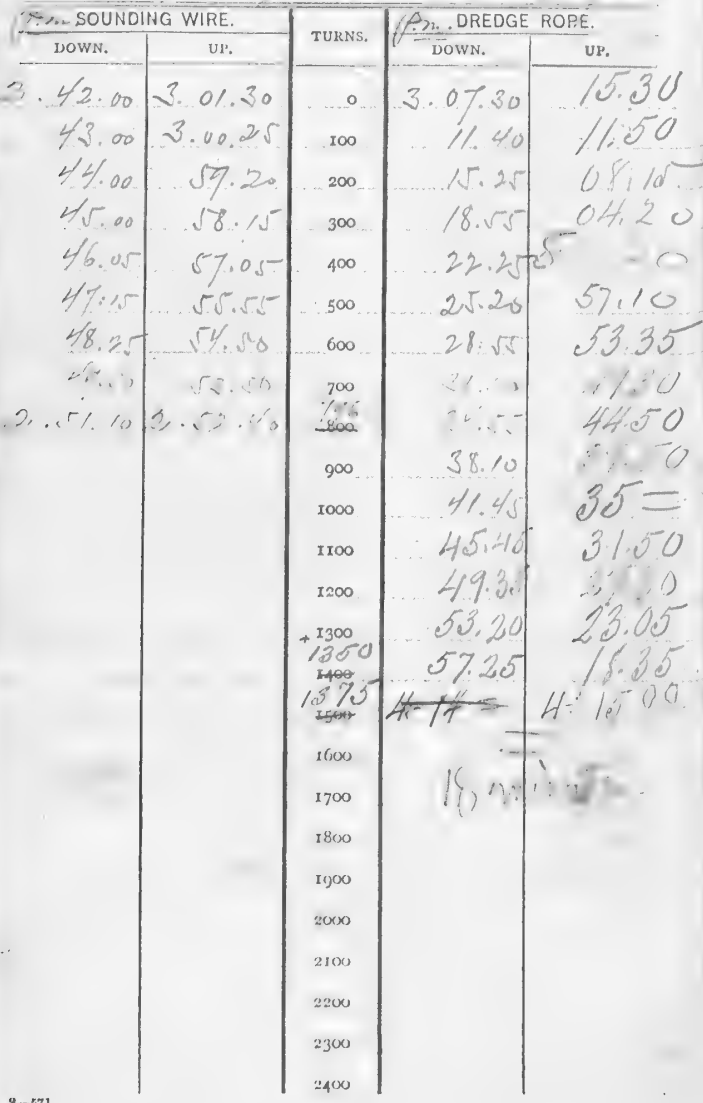


Centin of Brid $J^{\circ}-875^{\circ} \varepsilon_{1}, 11^{2} 10^{\prime}$

SERIAL TEMPERATURES.

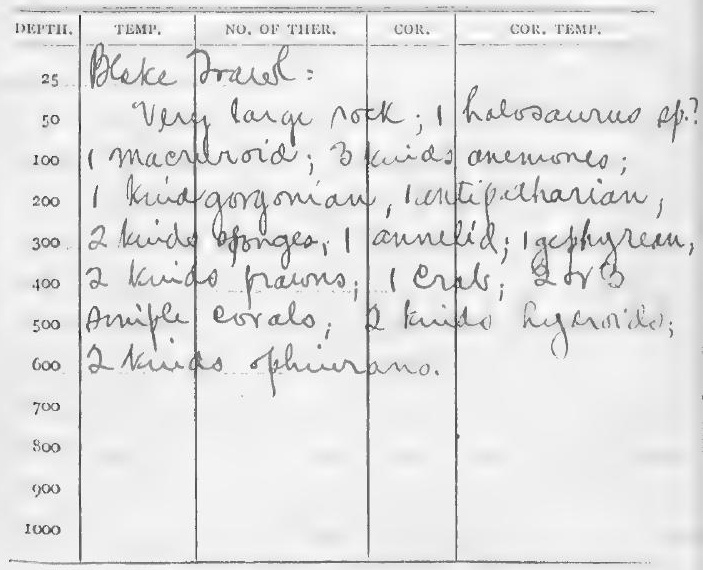

Thu REMARKS:

its 1375 fune. 


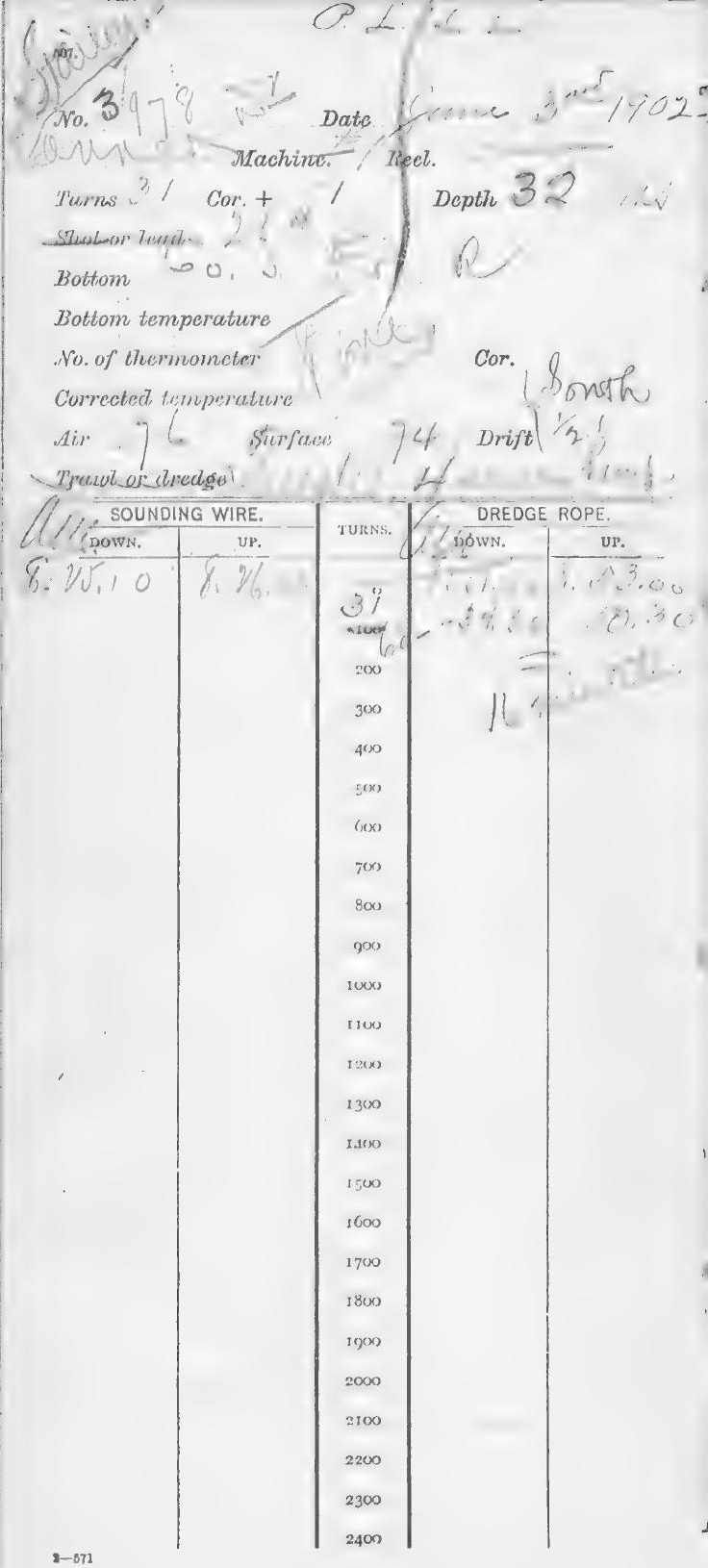


Guin of

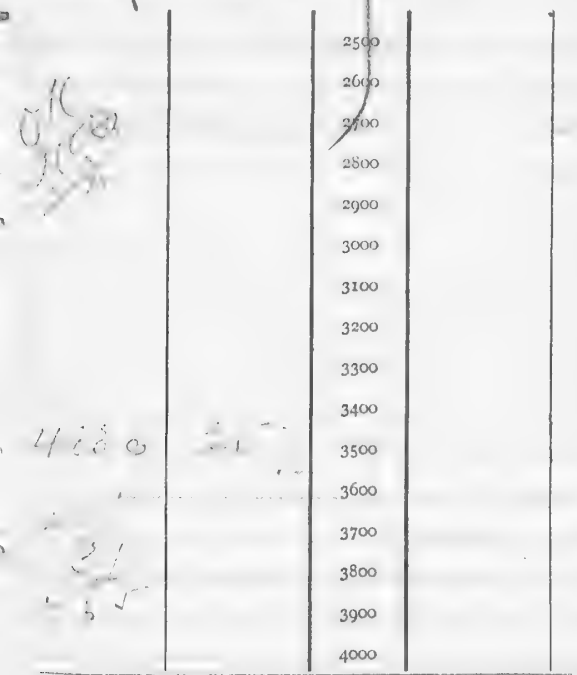

SERIAL, TEMPERATURES.

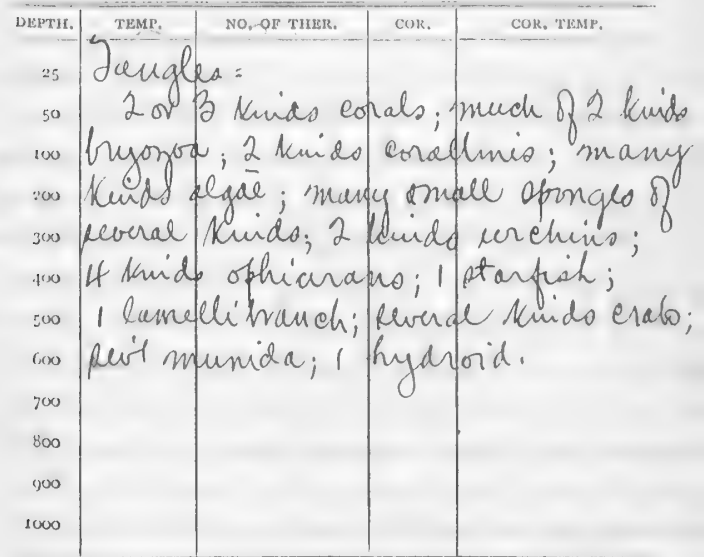

REMARKS :

nowerts 


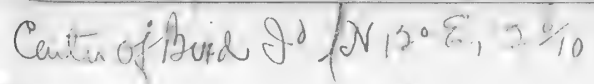

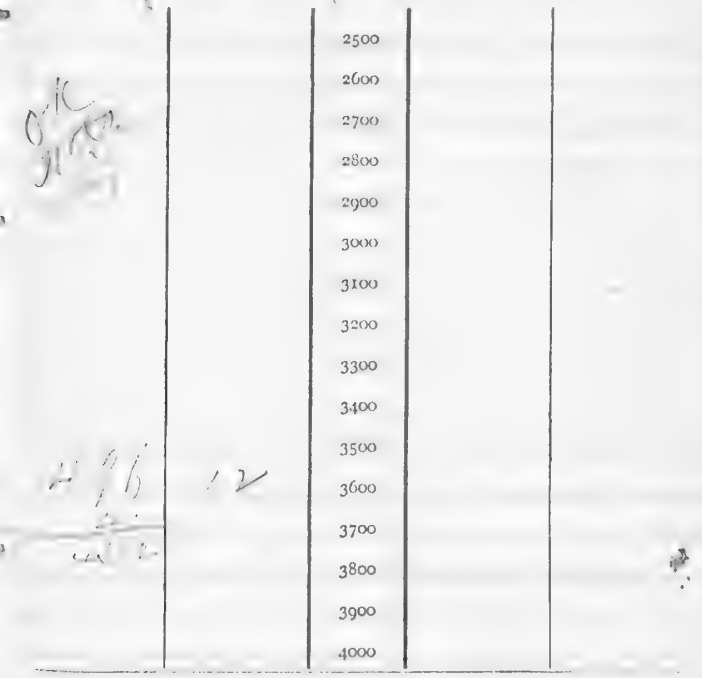

SERIAL TEMPERATURES.

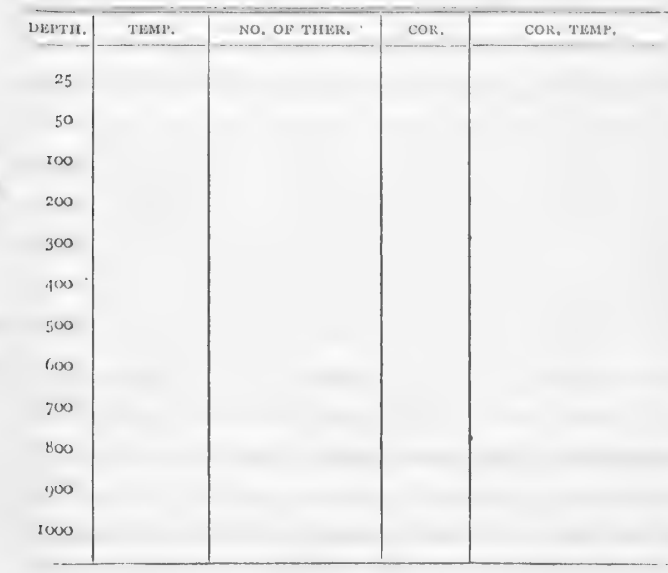

REMARKS : 
digntsil Machine. I' Reel.

Turns/c Cor. +

Depth $/ 0$ ?

sinot or lead

Bottom the

Bottom terihperature

No. of thermometer

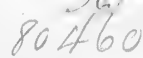

Cor.

Corrceted temperature
Air
Surface
Drift

Trawl/or dredge

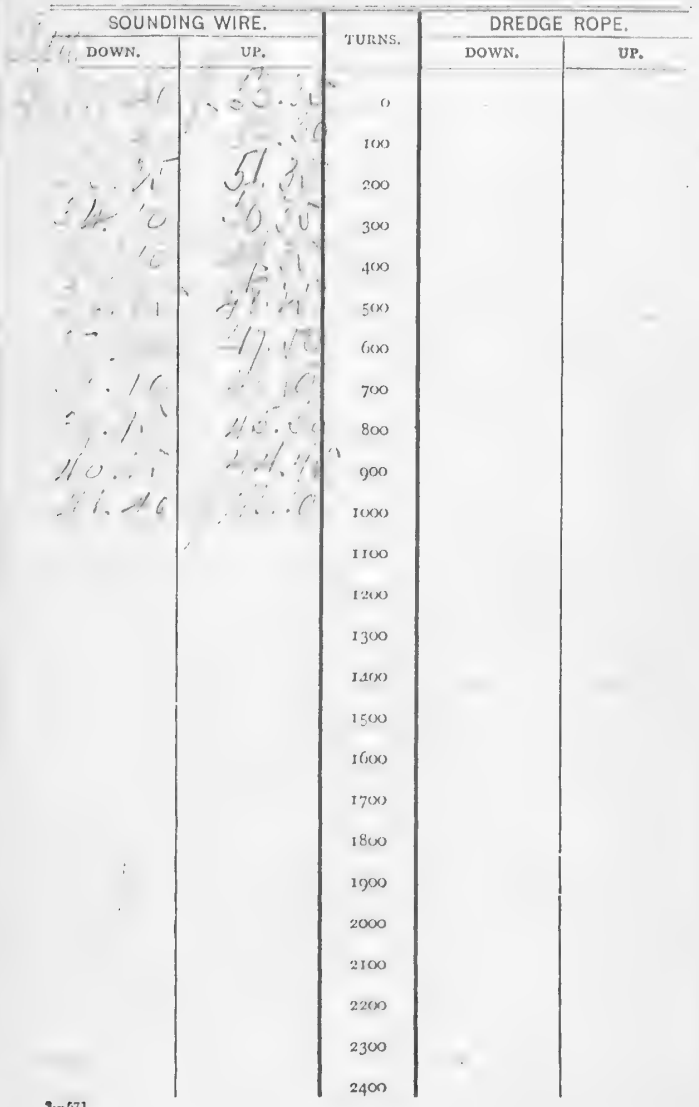




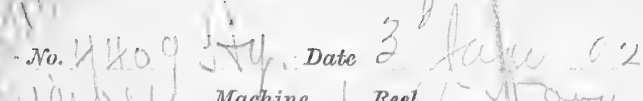
) 880 Machine. I Reel.

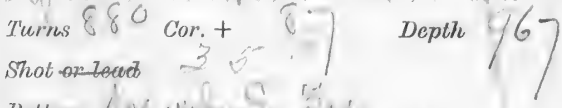

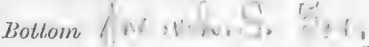
Bottom temperature No. of thermometer

Cor.

Corrected temperature

Air Surface

Drift

Trawi or diredse

SOUNDING WIRE.

(i)

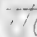
DOWN. $\therefore, 1 / 1 /(\cdots, i, j)$ $2,1,2$ , , $3,1,1,1 / 0$ $\therefore \quad i \quad 1 / 1 \%$ 1)... 4 $\therefore$$$
-
$$

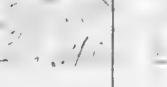

\begin{tabular}{|c|c|c|}
\hline \multirow{2}{*}{ TUKNS. } & \multicolumn{2}{|c|}{ DREDGE ROPE. } \\
\hline & DOWN. & UP. \\
\hline
\end{tabular}




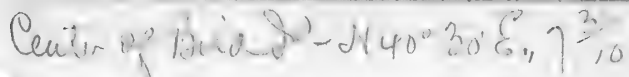

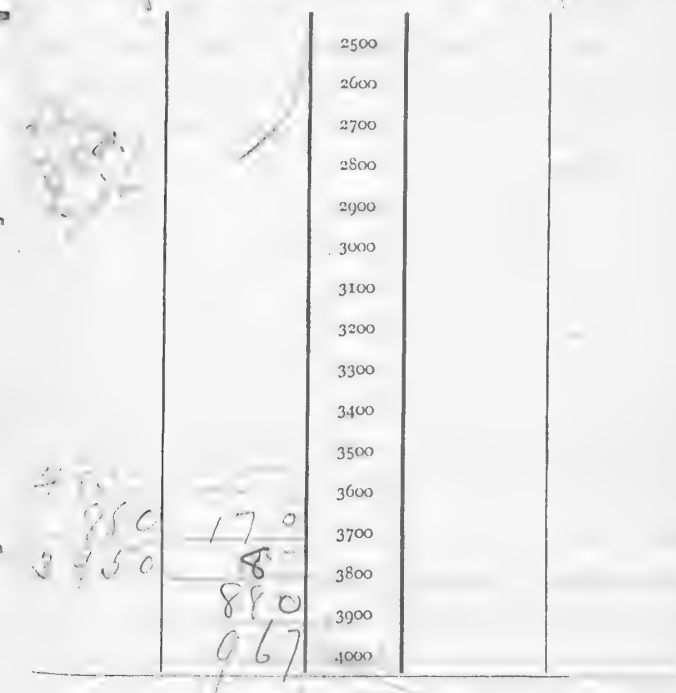

SERIAL TEMPERATURES.

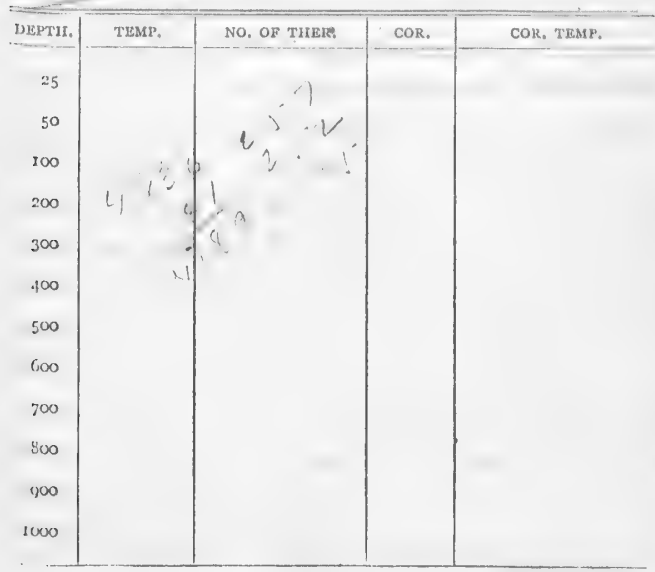

REMARKS : 
ion.

No. 1) I $110,(1)$ Date

II A i l I Machine. I Reel.

Turns 733 Cor. $+\ldots, 73$ Depth 806

Shot or-iead

Bottom fou wh. S. Jov.

Boltom temperature

No. of thermometer. 80460 Cor.

Corrected temperature

Air... Surface 74 , Drift

Trawt or aredge

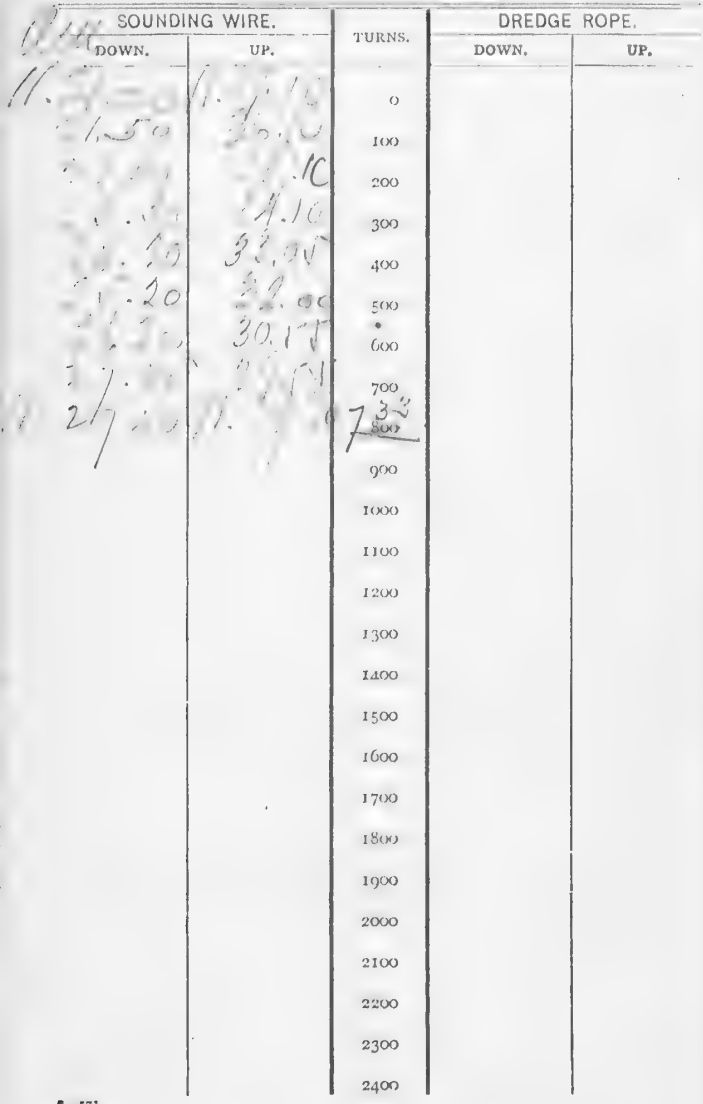


No.

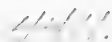

\section{Dute}

Ci, Machine. "', Reel.

Turns 2 Cor +

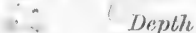

shot on-lared

Bottom freruh.

Bottom temperature

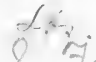

No. of thermometer

Corrected temperature
Mir<smiles>C1C2C3C4C1C1C2C3C41</smiles>
- Surface
$7 \sqrt{-}-$ Drift

Trawt or dredge

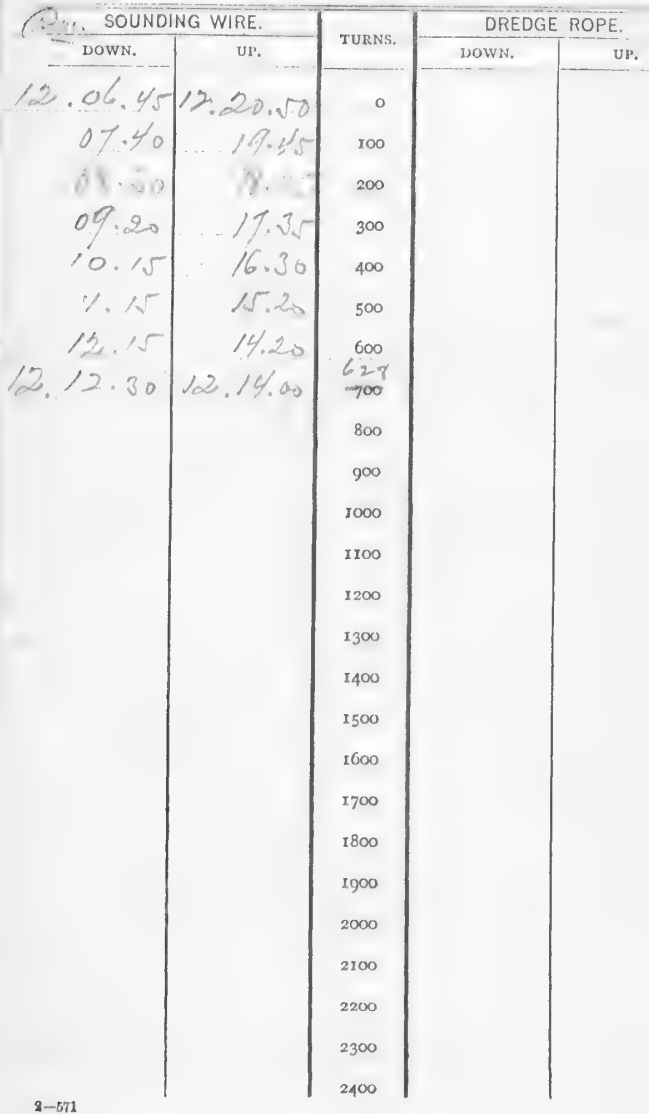





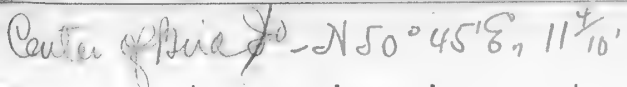

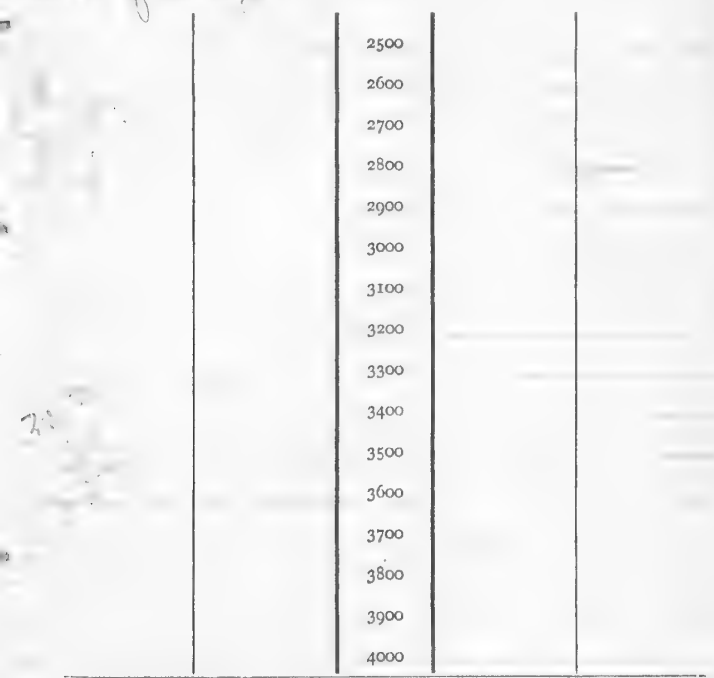

SERIAL TEMPERATURES.

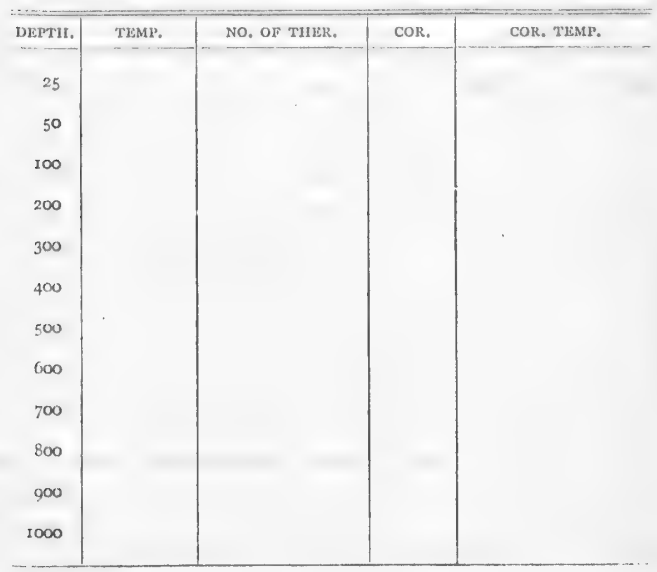

REMARKS : 


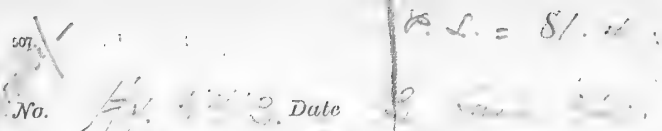

Ciras Machine I Recl.

Furms 2,5 Cor. +2 (Depth : Shot or bout is $\sqrt{6}$.

Bottonis is of a

Bottom temperature

No. of thormometer

$74:-$

Corrected temperature

Air 76. Surface 75 - Drift

Trawl or dredge

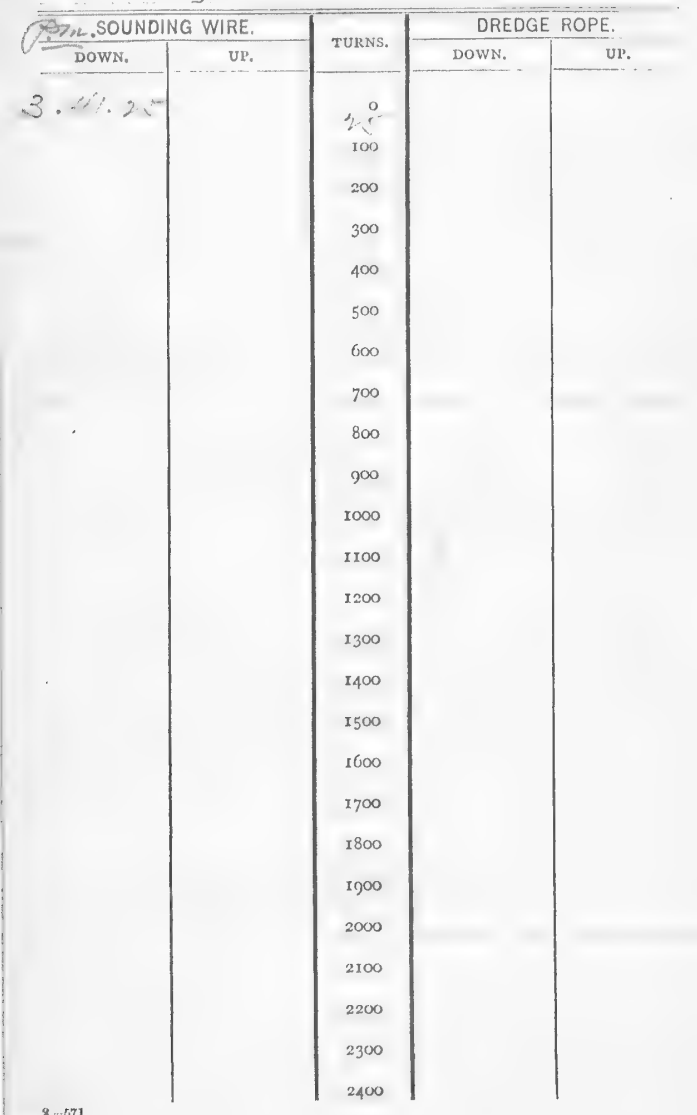




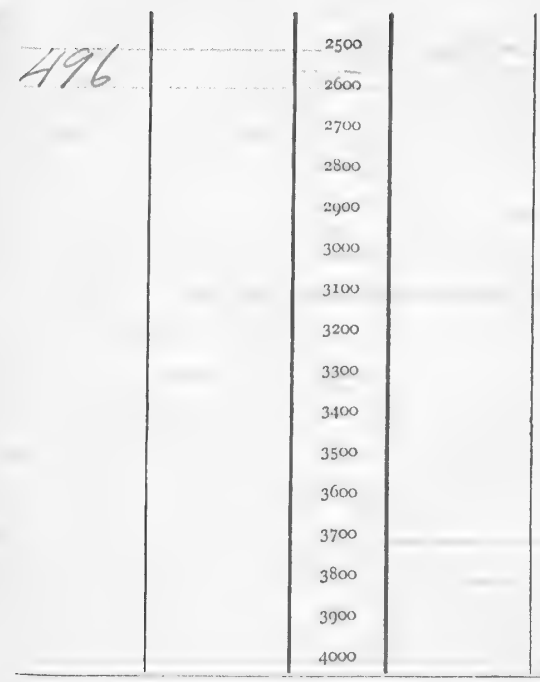

SERIAL TEMPERATURES,

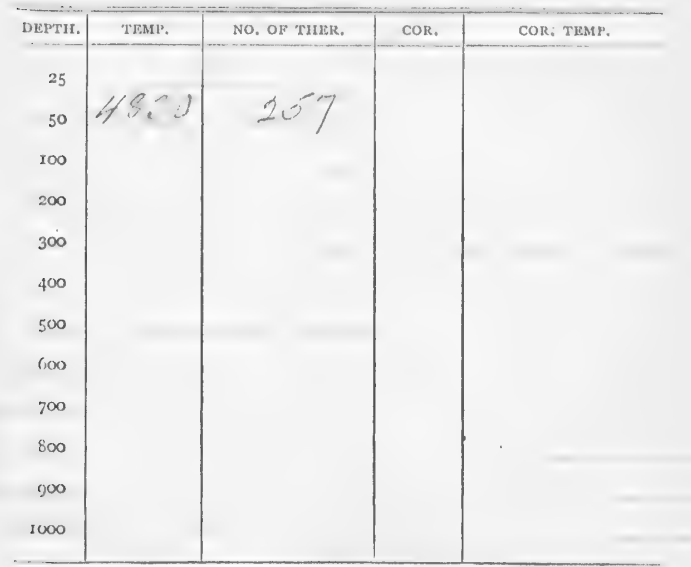

REMARKS : 


\section{Date

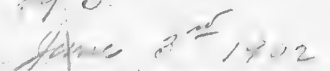

H. Machine." Reel.

Turns $3 / 7$ Cor +32

Depth

Shot an tored

Bottiom

fue bo

, $S_{0} T_{0}$

Bottom temperature

No. of thermometer

80460

Cor.

Corrected temperature

Air $7 f$ Surface Drift

Trawl or dredge

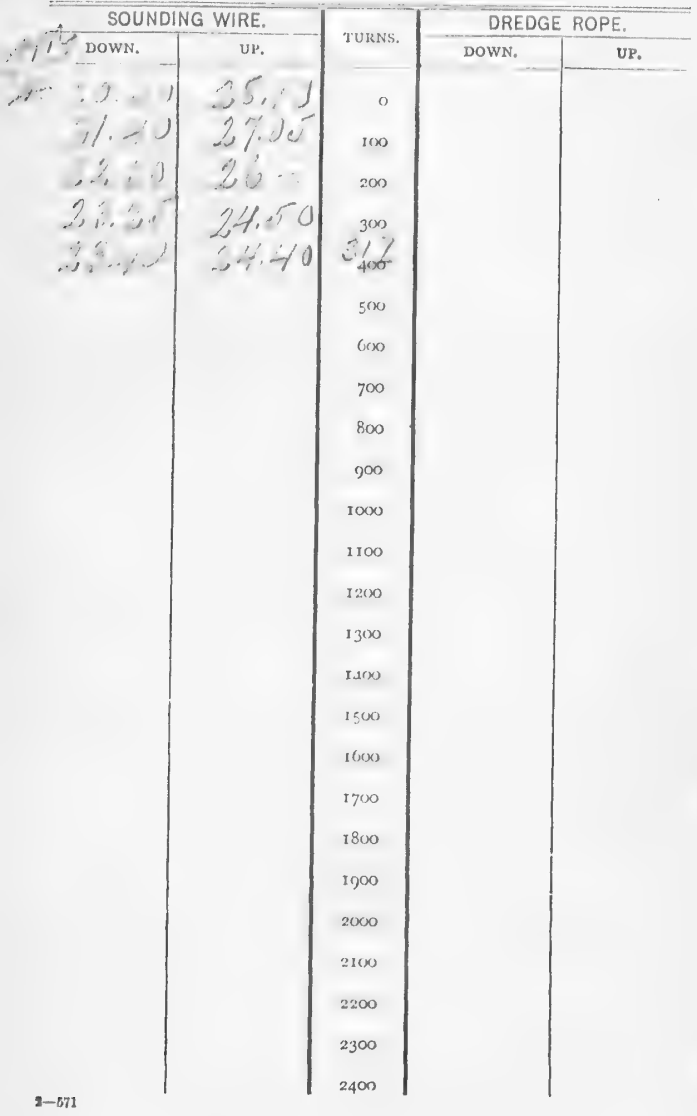




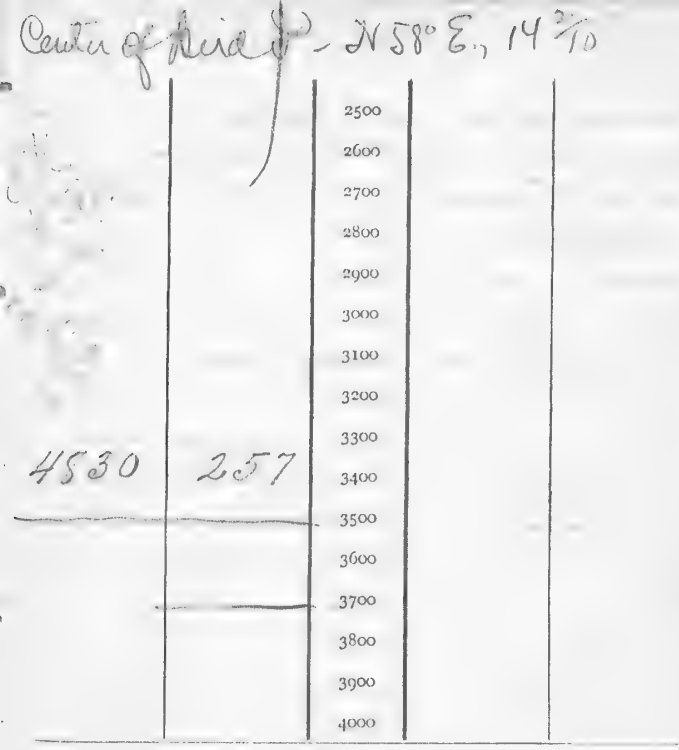

SERIAL TEMPERATURES.

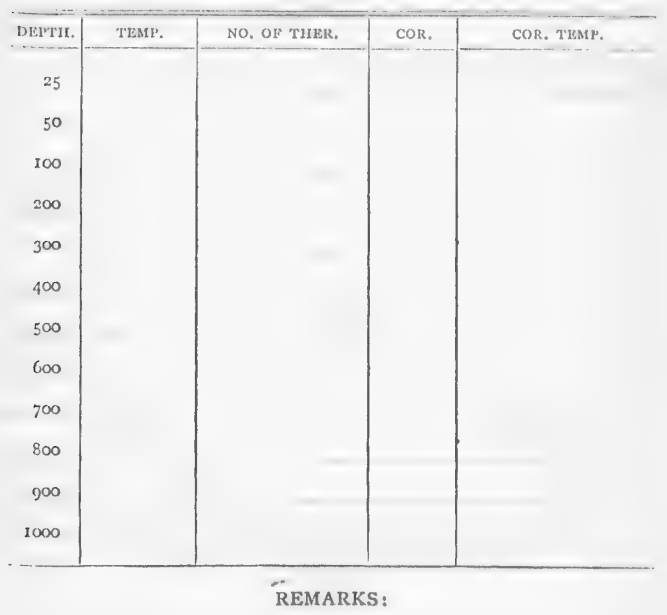




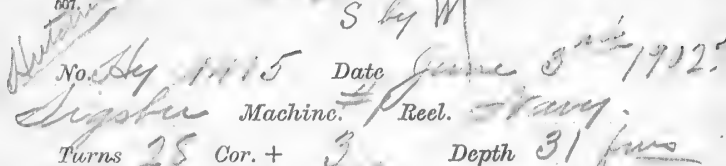

Turns 25 Cor.t, Depth 3/ fino

Bottom

Bottom temperature

No. of thermometer

Corrected temperature
Air
Surface
Drift

Trawt or dredge

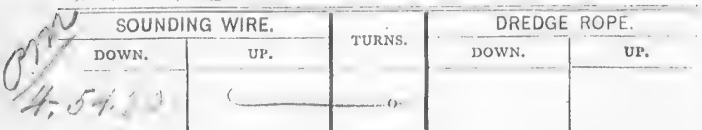




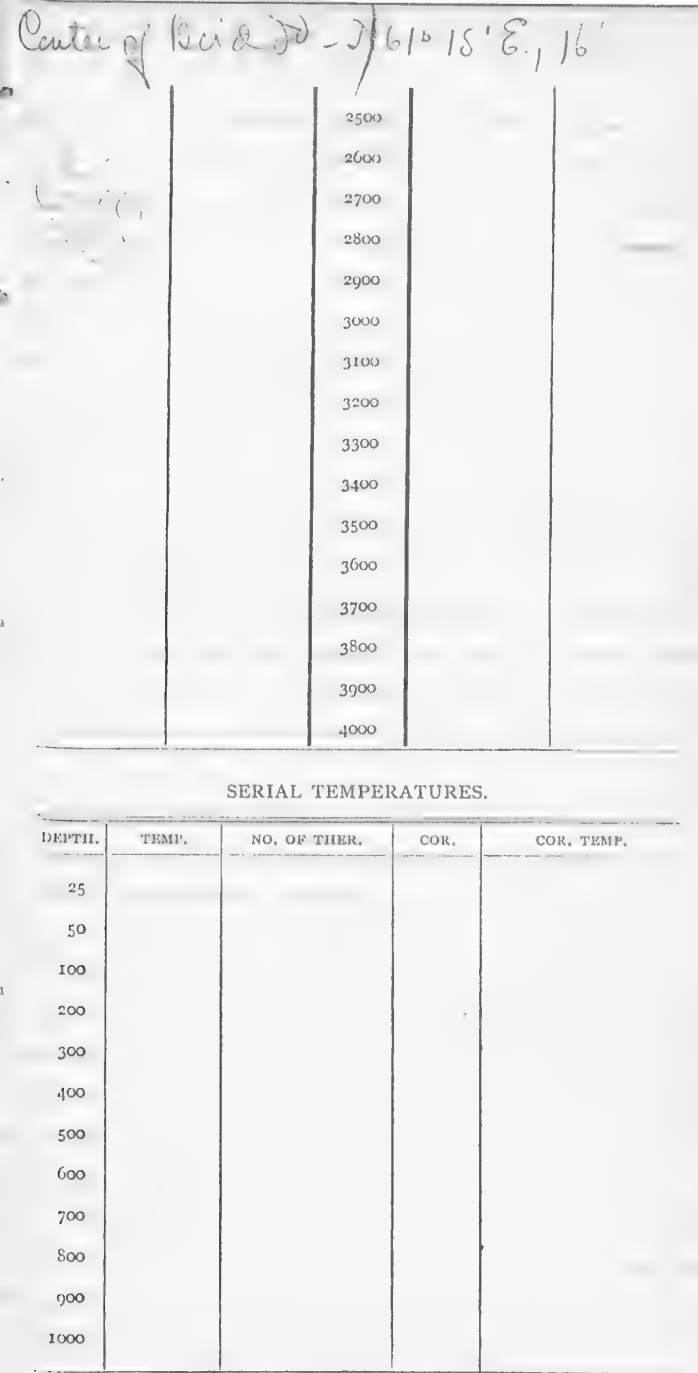

SERIAL TEMPERATURES.

REMARKS : 
Bottom tempdrature No. of thermometer \& 460 Cor.

Corrected temperature Air If Surface. 70 Drift

Trawl or dredge

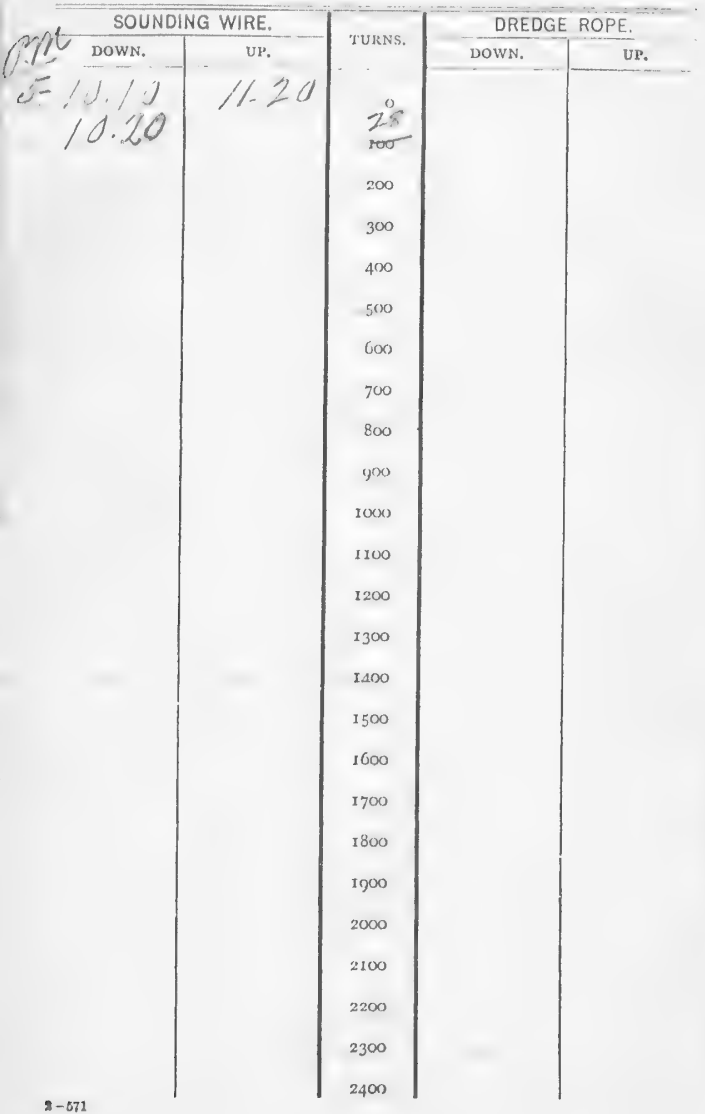




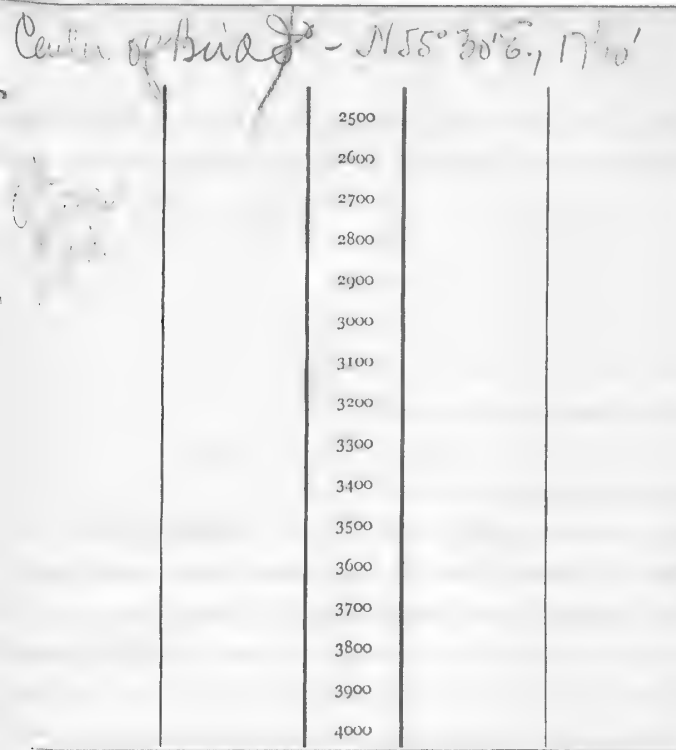

SERIAL TEMPERATURES.

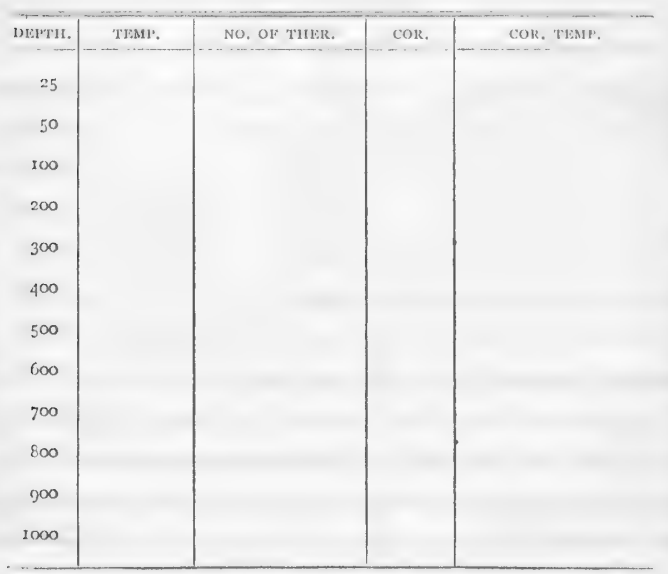

REMARKS : 
- porti

No.

Date

WN ( \& ') Machine I Reel,
Turns
Cor. +
Shat or lead
36 \%
Depth

Bottom $\mathrm{Ne}$. $\mathrm{C}$.

Bottom terriperature

No. of thermometer

Cor.

Corrected temperature
Air

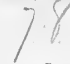
Surface
Drift
Trawt or dredge

SOUNDING WIRE.

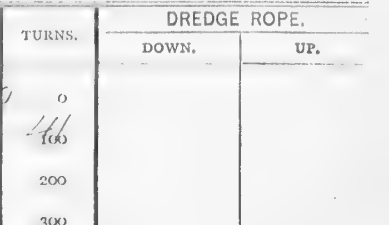

1700

I 800

1000

2000

2100

2200

2300

2400 


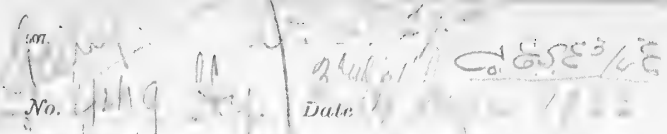

Machine. | Reel.

Turns 2/3:Cor + $/ 1$ Depth

Shotorlead $100 \div$

Bottom coe tes of ल⿸厂冫

Bottom temperature

No. of thermometer

Corrccted tempgrature

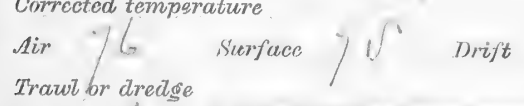

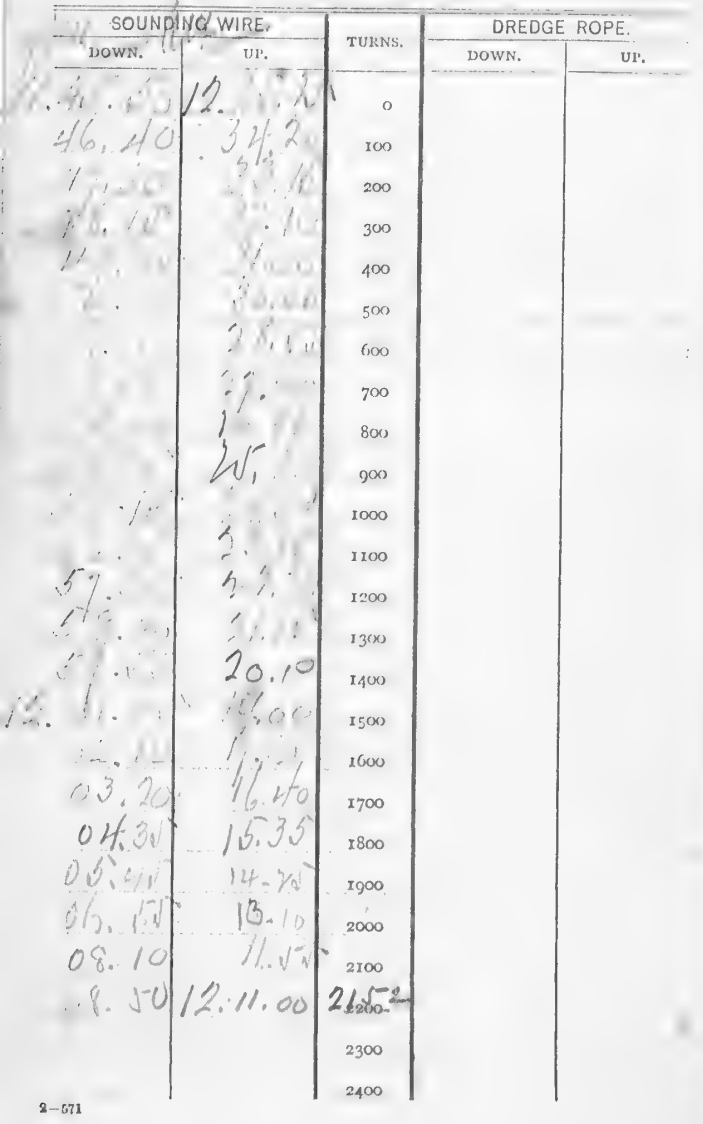




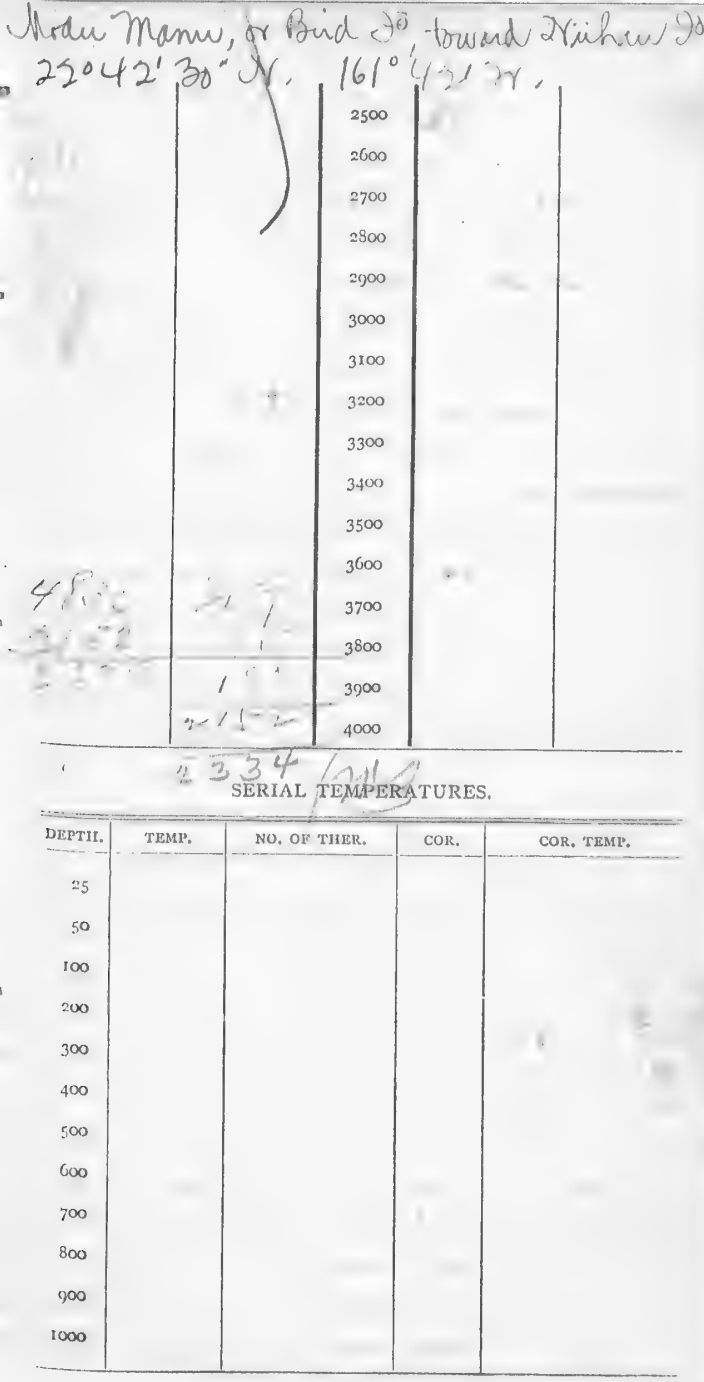


207

L.s.

No. $74 \therefore$ Date

"'. Machine. Reel.

Turns 2, Cor. +

Depth

shot on-lout Coe $\mathcal{L} !$.

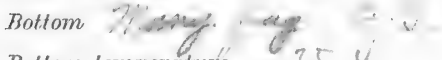

Bottom temperature $35: 4$

No. of thermometer 80460. Cor.

\section{Corrected temperature}

Air 7\% - Surface . 75. - Drift

Trawt or aredge

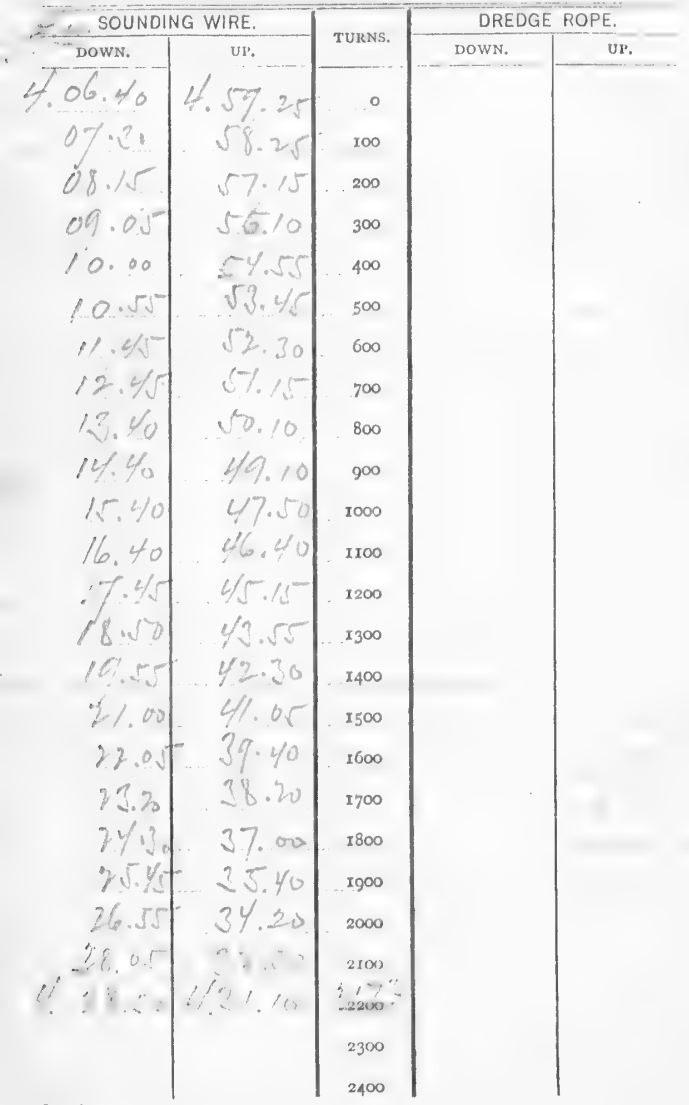




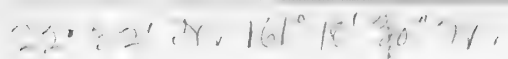

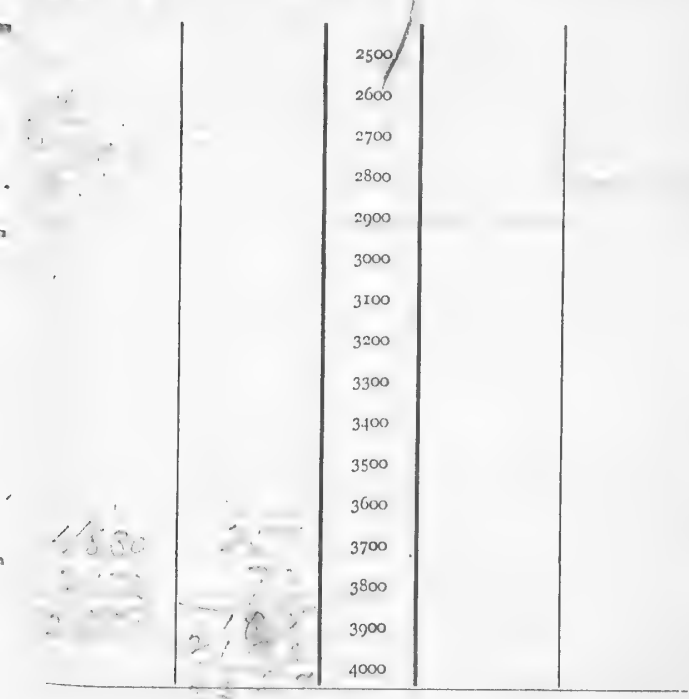

SERIAL TEMPERATURES.

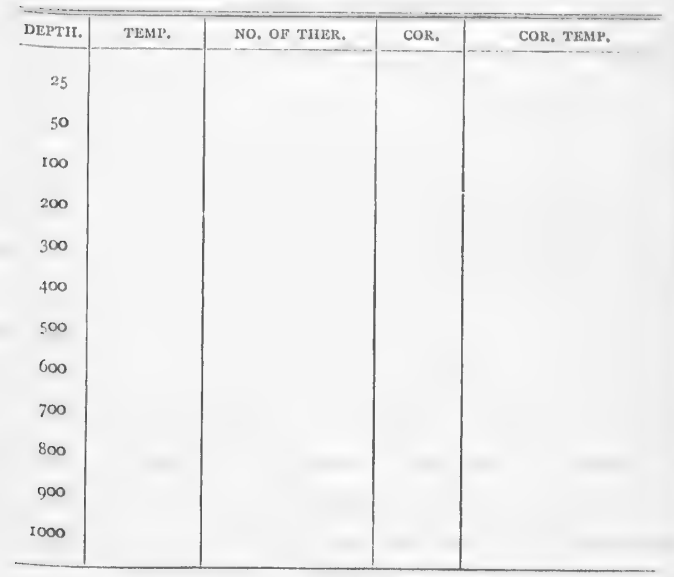

REMARKS : 
Wo. H4 I Date $\because \frac{1}{i} ;=;$,

Turns .. Cor. 786 Depth $2 \%$

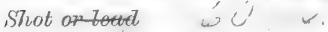

Bottom $17 \%$, . 1 . .

Bottom temperature $35.5=0$

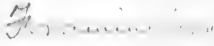

No. of thermometer 5 if 60 Cor.

Corrected temperature

Air 75 strface 76 Drift

Trawt or dredge

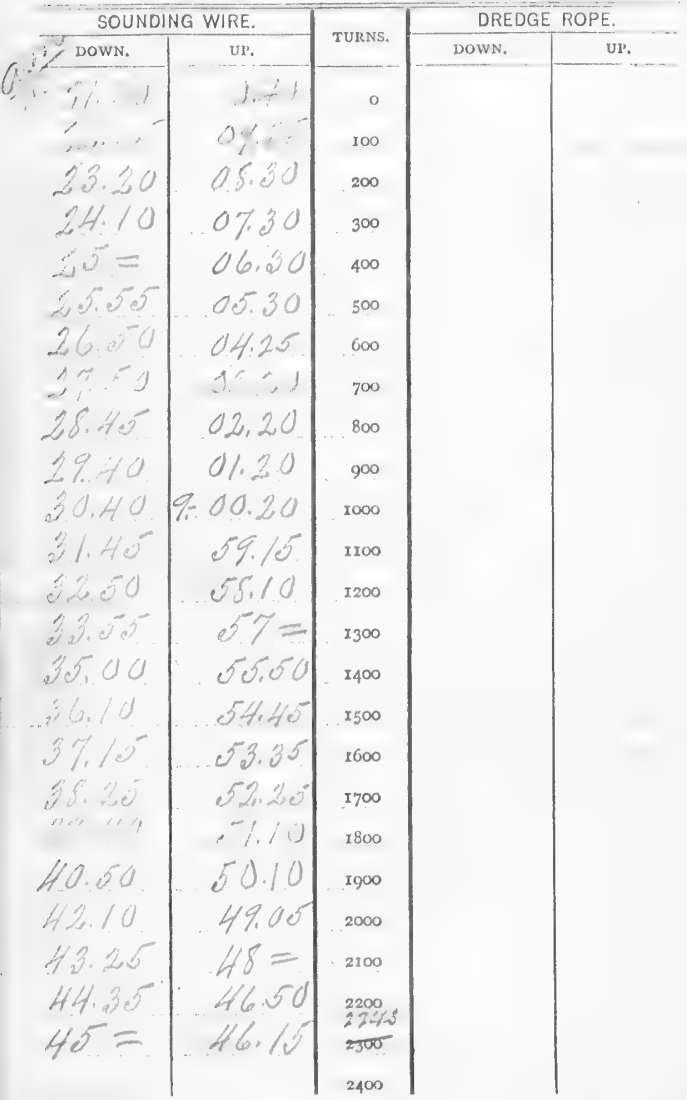




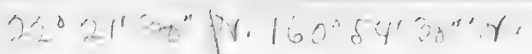

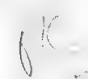

SERIAL TEMPERATURES.

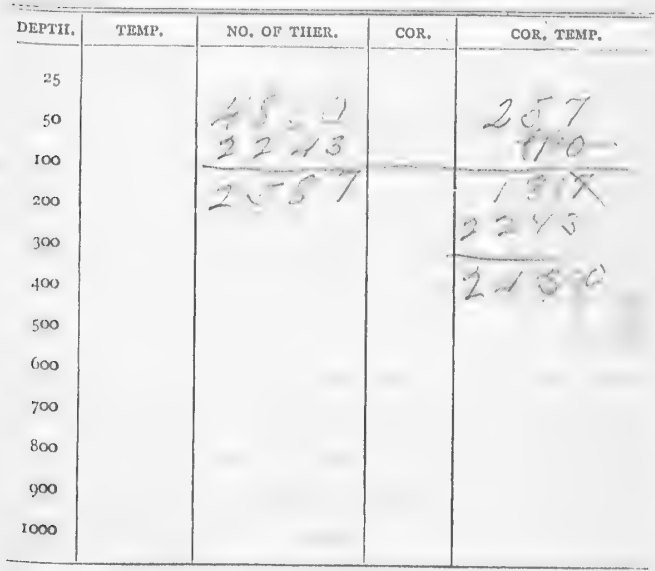

REMARKS : 
Turns 1686 Cor +165

Shot on teach

\section{Bottom}

Bottom temperature

No. of thermometer

Cor.

\section{Corrected tomperature}

Air ?7 . Sterface .76 ... Drift

Trawl or dredge

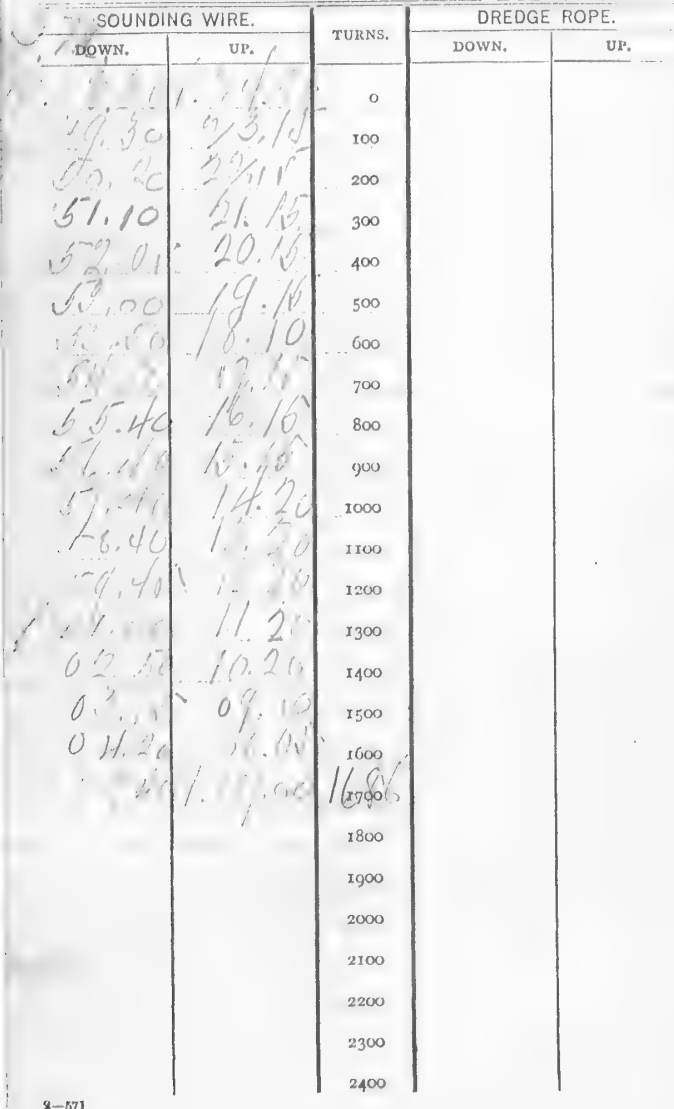


$22011^{\prime} \partial V_{1} 160^{\circ} 30^{\prime} 30^{\prime \prime} \gamma$.
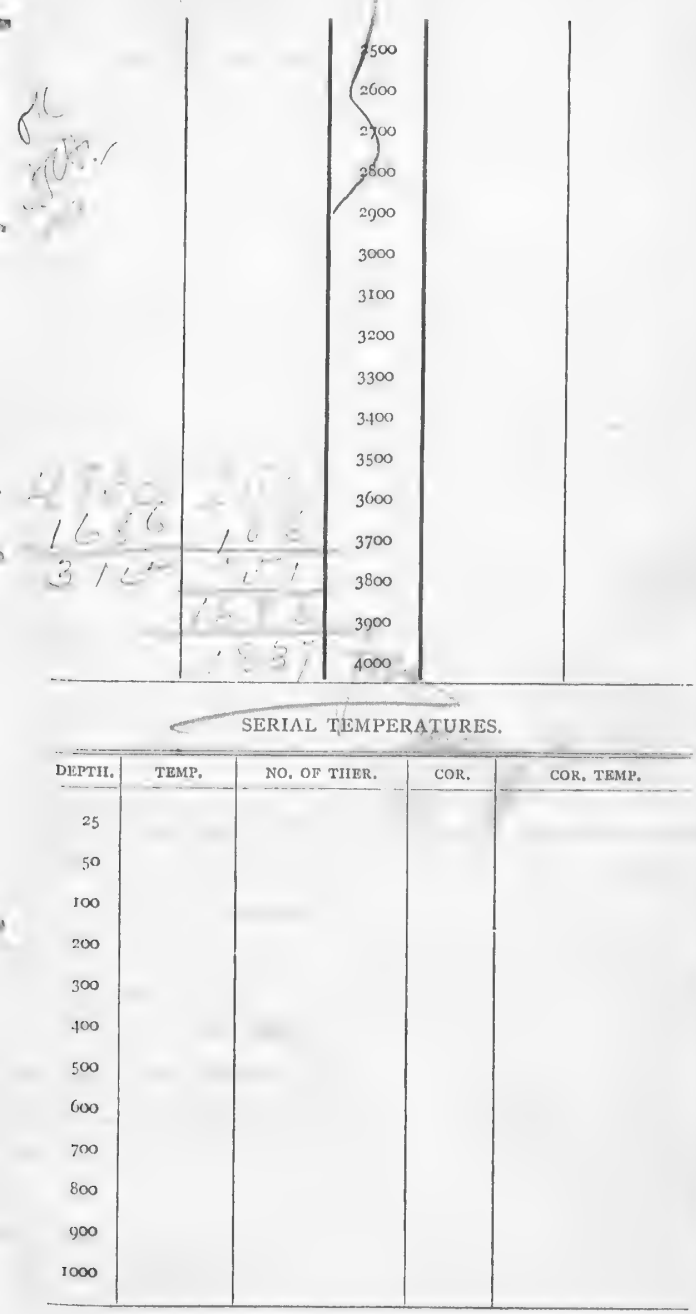

REMARKS : 
Turns $\quad$ Cor. + Machine.

Shot or lead

Bottom

Bottom temperature

No. of thermometer

Corrected temperature

Air - Sicrface.

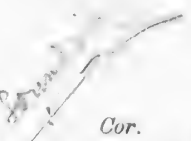

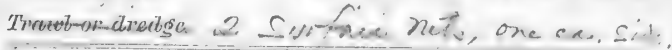

SOUNDING WIRE.

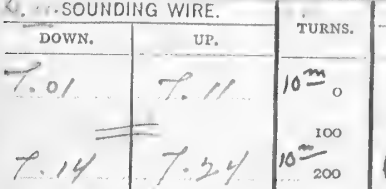

\begin{tabular}{c|c|c|}
\hline DOWN. & UP. & TURNS. \\
\hline 7.01 & 7.11 & $10 m_{0}$ \\
\hline 7.14 & 7.34 & $10=\frac{100}{200}$
\end{tabular}

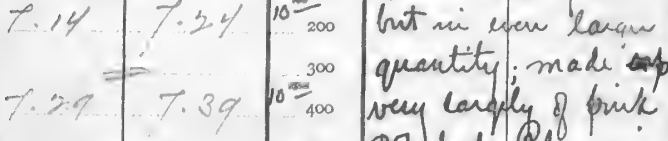

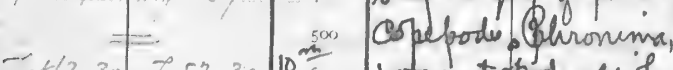

$\because 42.30 \quad 7.52 .3010 \frac{6}{600} 1$ atmatoporl, wil

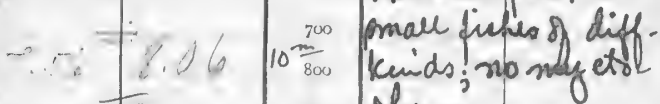

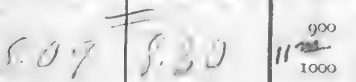

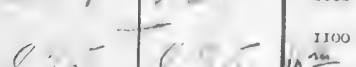

$8.20^{-} \int: 2 e^{-} 10 \frac{320}{1200}$

1300

7 racels $=71^{\text {new }}$.

Spit hayl:

Carqu apantity 8 ioco efepods; ieveral tos * timare meduoae $\sigma$ otmabhol. íngl. ore 2 omall frthe ; tur sforda: halotites: Dogitai, cilt if $\{$; a four cleb lavati pul of 2300 cristaces; muclu of haers. the

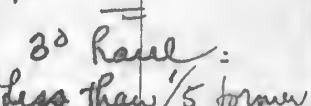
Hess thaw $1 / 5$ forour amount, int no other decided changer 1 frie heftur opod. $4^{\text {th }}$ Roul.

Qlewort nothing as to quantity, Copepolo

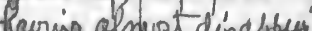
vering alnuat desappes. I hipporoupas: lqu heteroposto; glest fiche; Lew my fophum; ctenothathes iremg mumucoso. 


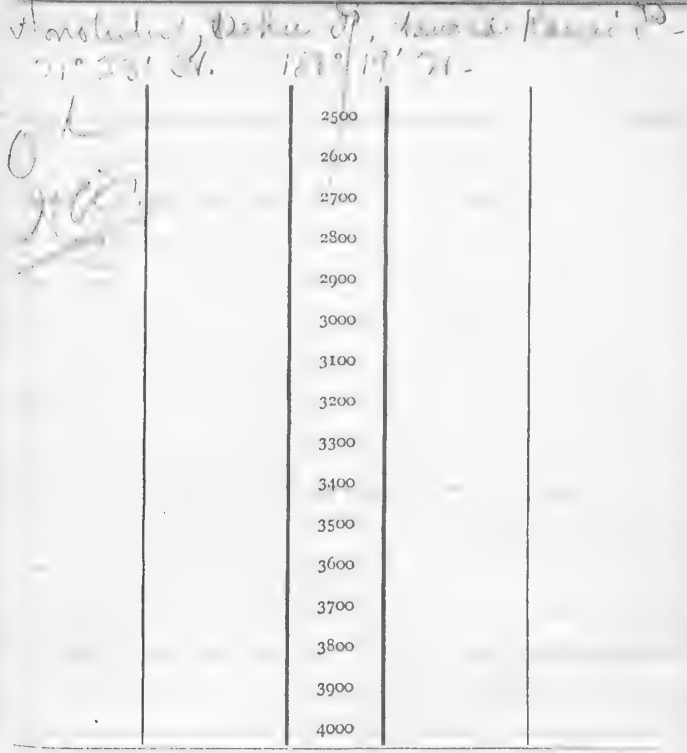

SERIAI, TEMPERATURES.

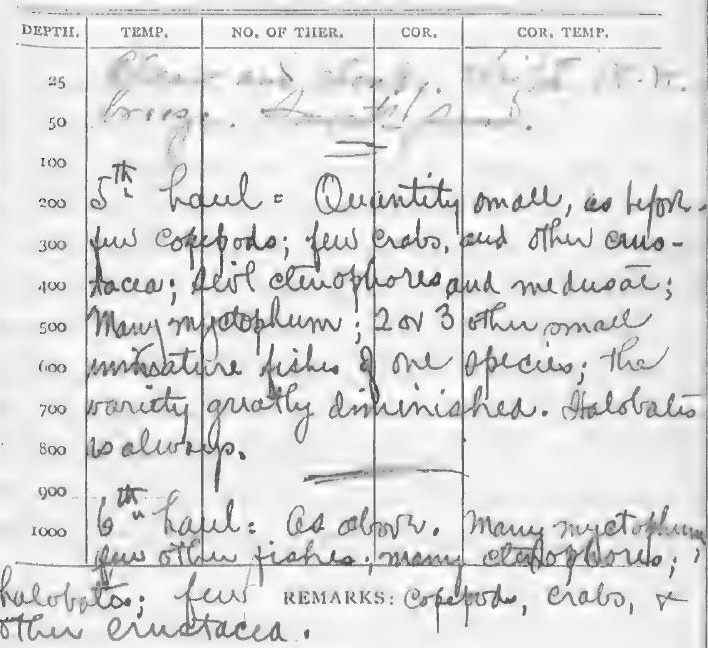

7t Kaul. as befor's. The mass of mativial made up is in Racels 4-f

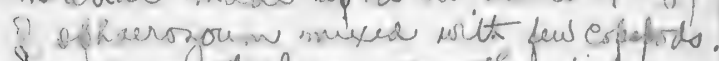
y aral maxctophuin; no oflat iche. Mung ctinophotes; o sempilide. 
(.) \& I, Machine. / Reel:
Turns 59 ? 6 Cor. +58
shot or lead...
lopth $60^{36}$ pos

\section{Botlom}

Bollom temperature

No. of thermometer

Corrected temperature

Air Surfaco

\section{Trawt-or dredige}$$
\rightarrow 7
$$

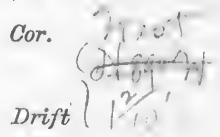

$+\infty, \ldots+\infty$,

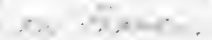

PI SOUNDING WIRE:

Eubown.

\% 80

- $\therefore 0$

$\therefore \therefore-10$

$\because 1, \pm$

$\therefore 20$

$\therefore \ldots$

$\because \ldots 2$ UP. TURNS. $(1, i, \cdots, j)$

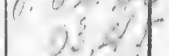
$0 \div, 1,1$ O.:. 0 i) 1,0 1:00.00

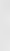

$\therefore$ $8 x$ 8.2 Gre

8.44 8.40

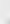

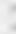
$1.40-20-3.59$ * reped ant ds 710 theी Rcamy 90

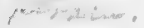

2-871

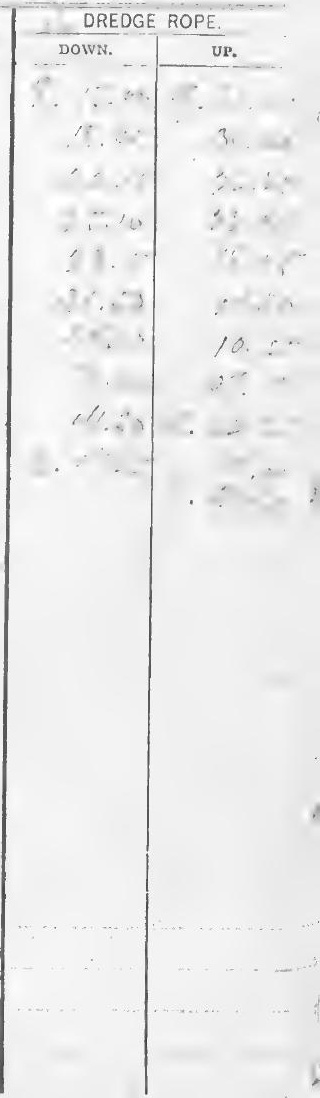


Vieinit of Kauai

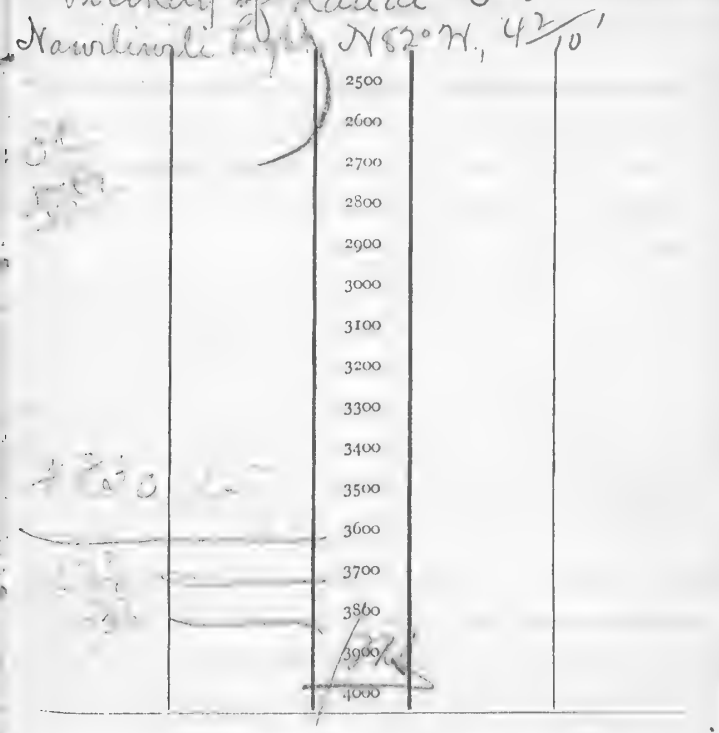

SERIAL TEMIPERTURES.

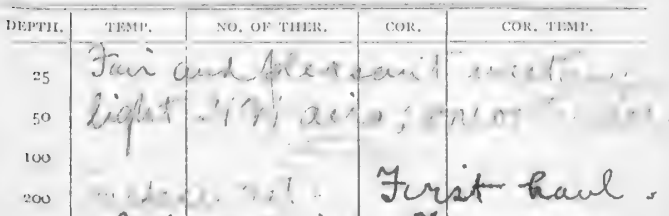

$3(x)$ hitte mativial. Fus somace +10 phore fishes of kmid. that peem 500 mot tho be att parface at night. coo tees karg blue espefods and sther 700 Presitaper, Ralobates; epharrourn;

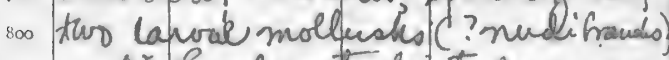
goo 20 haul not lested.

Dangle = Pord haul with muoh branching gorgoniano + ophicrano pound abbut them. If kinids ophicenavo; I kind otarfich; munida; dermit; isotol; sponge; herchin; gonostoma; 2 knido gorgoniano. 
$\mathrm{m}$.

No. Date $10,02$.

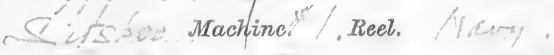

Turns $3 \%$ Cor. +3

Depth

Strobor lead 3L Lbs.

Bottom mine tore ce se

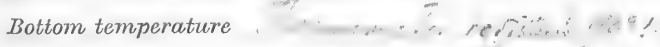

No. of thermometer $5048 \%$. Cor.

Corrected temperature

Air .. 86. Surface . 7\%. Drift

Trawl or dredge

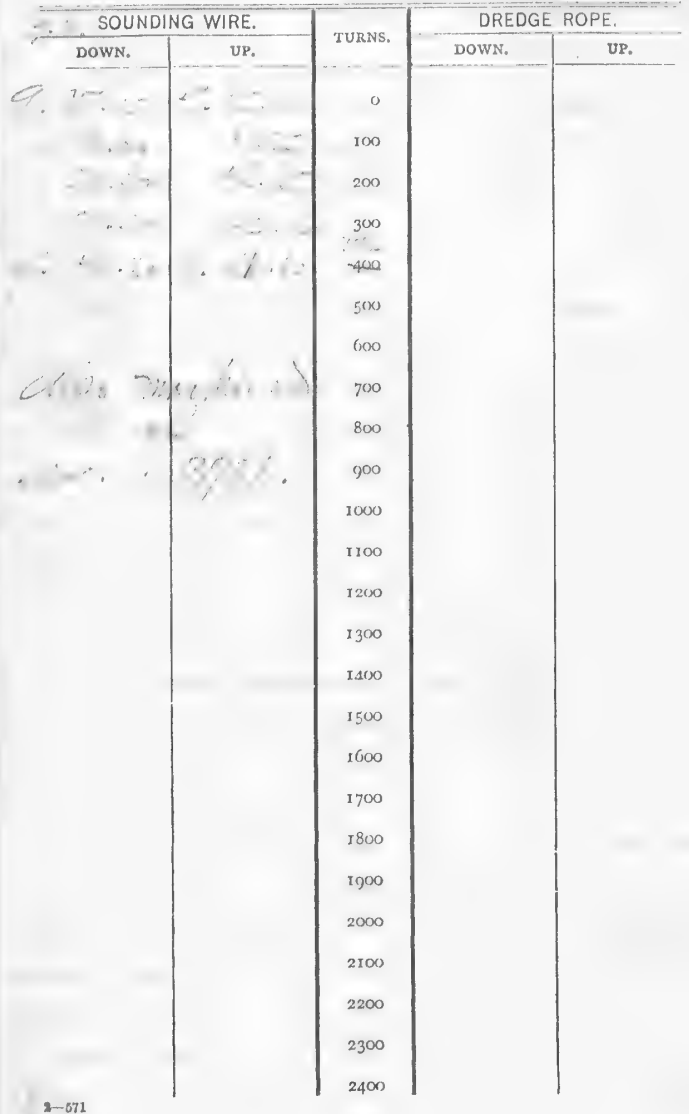


Machine.

Reel.
Turns 212Cor.
Depth ; $\because \cdots$.
Strotor lead
$\sqrt{6}$
21
Dopth a $=$ "

\section{Bottom}
Bottom temperature
No. of thermometer
$2 \therefore$ Cor.
Corrected temperature
Air 84. - Surface

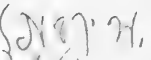 $\int 01,1$
Drift

Trowet-or.edredge

- SOUNDING WIRE. DOWN.

$\because$

$\therefore, \because$

, $\cdots$

,$\cdots$

UP.

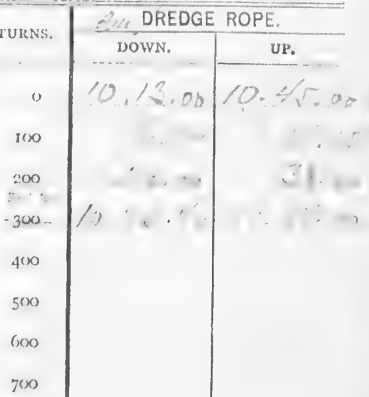

800

900

1000

I I 0

I 200

1300

I $\triangle 00$

1500

1600

1700

1800

I 000

2000

2 IOn

2200

2300

2400 


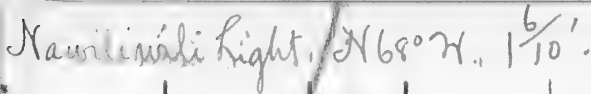

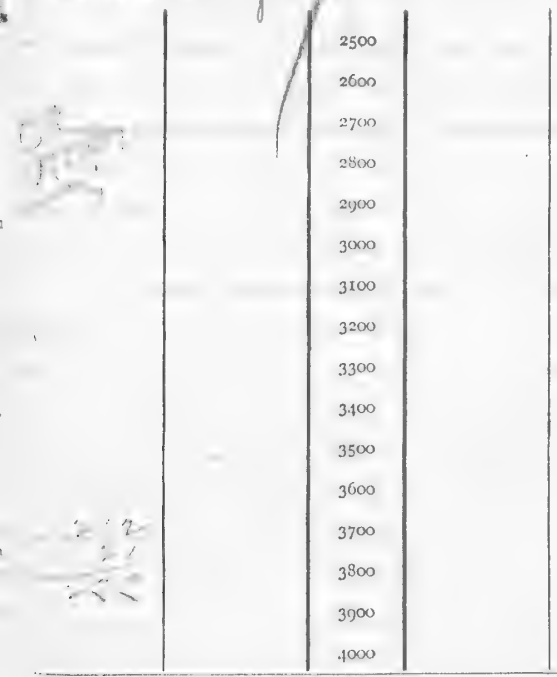

SERIAL TEMPERATURLS.

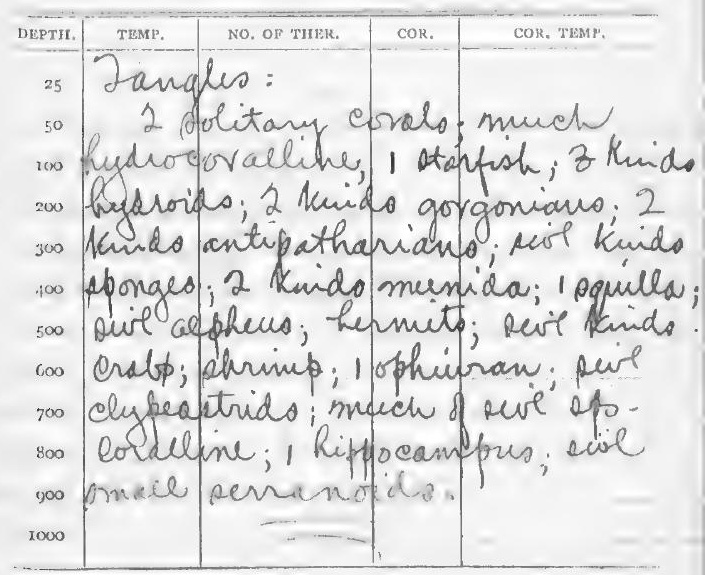

REMARKS : 
m.

'No. $44 / 24$ Date

S. Machine Reel.

Turns 1 . Cor.

Shat-on lead

Bottom cra śres.S. S.L.

Bottom temperature

No. of thermometer Cor.

Corrected temperature

Air S\% Surface ... - Drift

Trawl or dredge

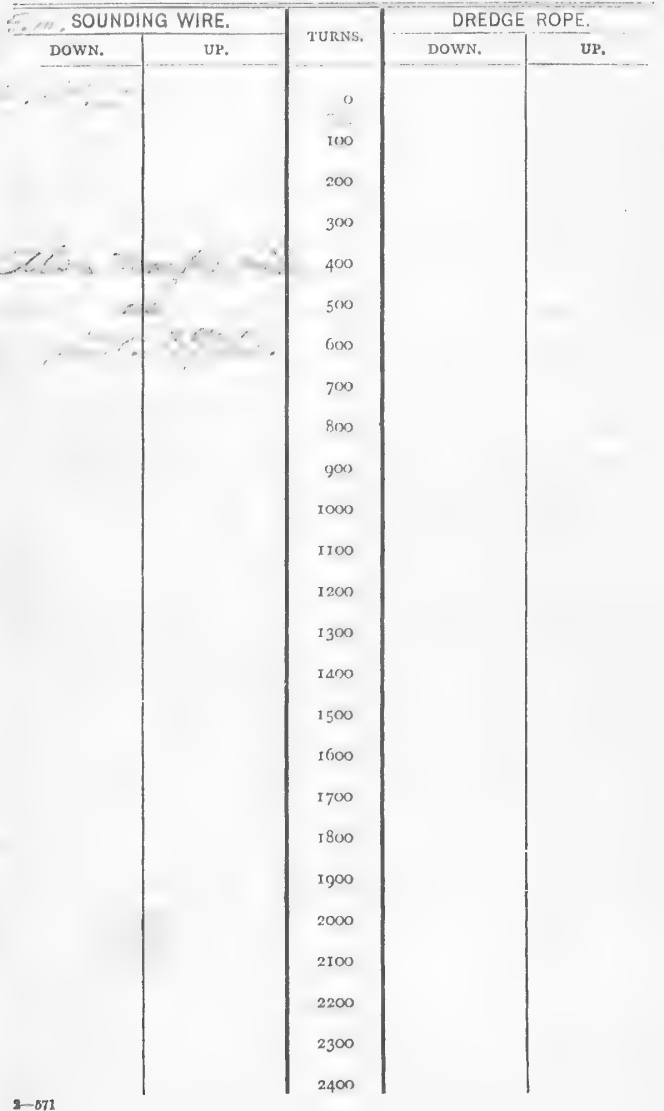




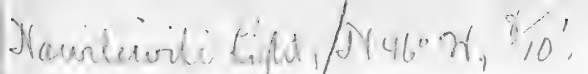

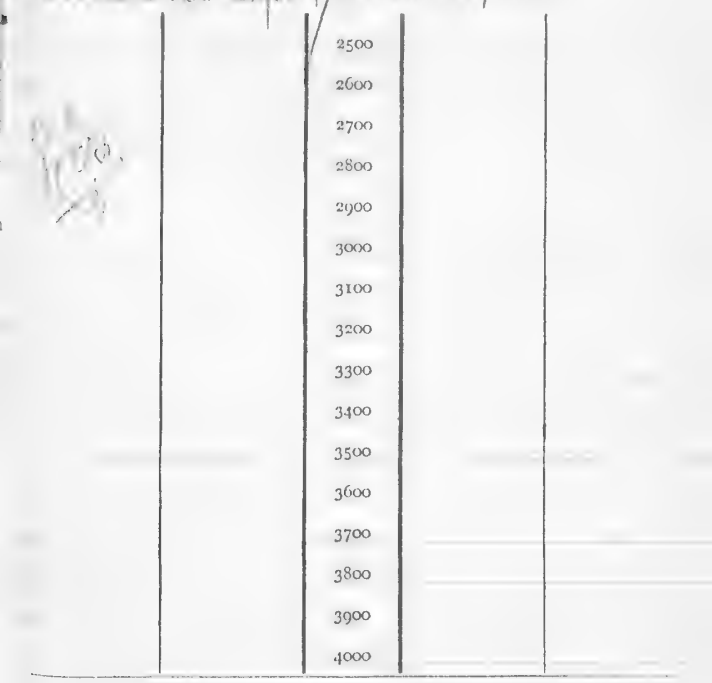

SERIAL, TEMPERATURES.

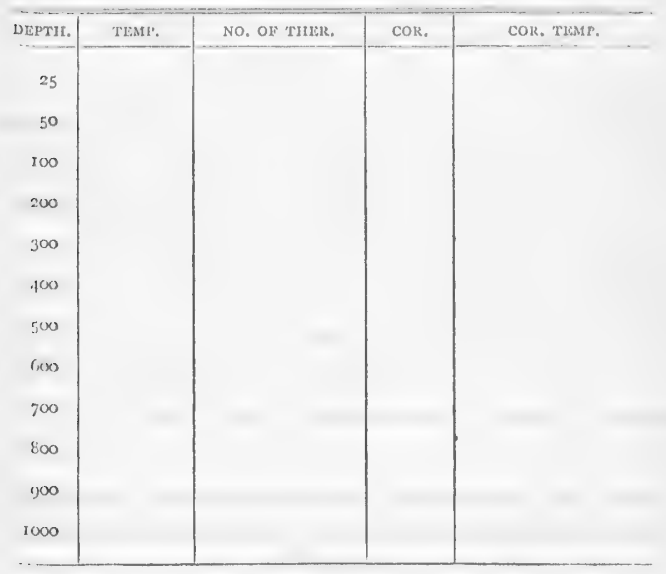

REMARKS : 
min.

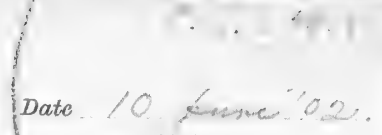

- Maching. / Reel $/ C_{2 m}$

Turns

Depth

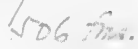

S7uot or lead

Bottiom

Bottom temperature

No. of themnometer

Corrected temperature

Air surface

Trawl of-ctrethe

1

Cor.

$\{y(5,7) \sqrt{ }$ Drift $2 \%$ -... SOUNOING WIRE.

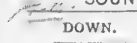

$11.34 \cdot 4 \sqrt{1} \cdot 4 \%$

(i)

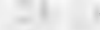

.

71

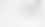

2-871

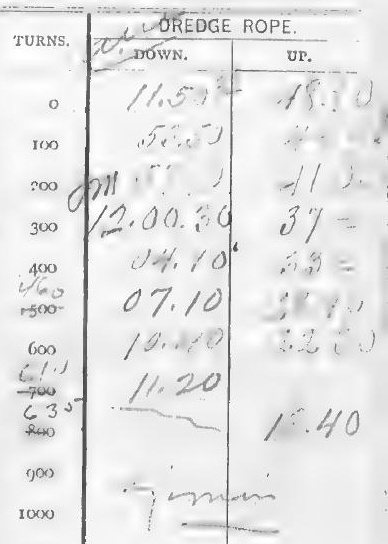

x 1 ก0

12100

1300

I. $10(x)$

1500

If(io)

1700

1800

19on

2000

2100

2200

2300

$240 n$ 


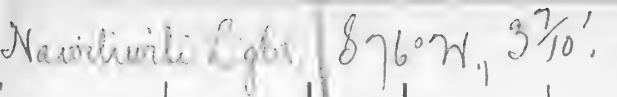

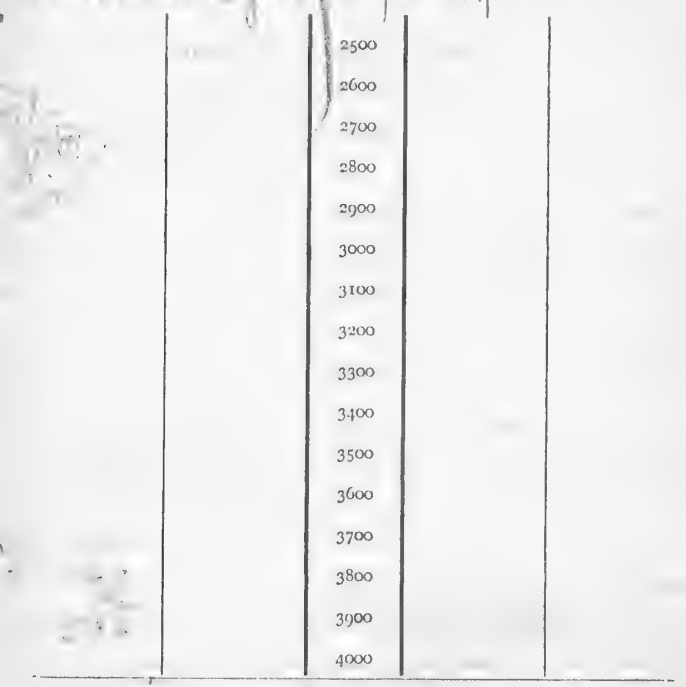

SERIAL TEMPIERTURES.

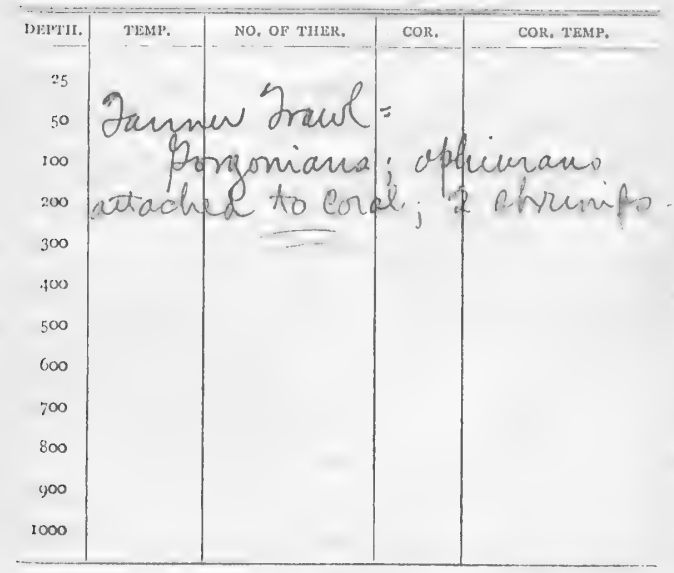

REMARKS :

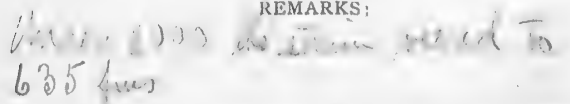

dit b, apsinat, hide sili.

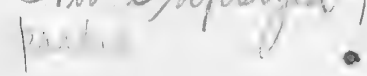




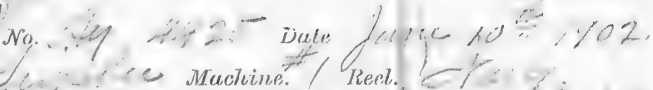
Turns 344 Cor. +54 Depth

Shotior lead

Bottiom

$$
26
$$

Bottom temperature

No. of thormometer.

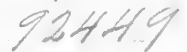

Cor.

Corrected temperature
Air
Surface

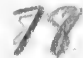
Drift

Trawl or dredge

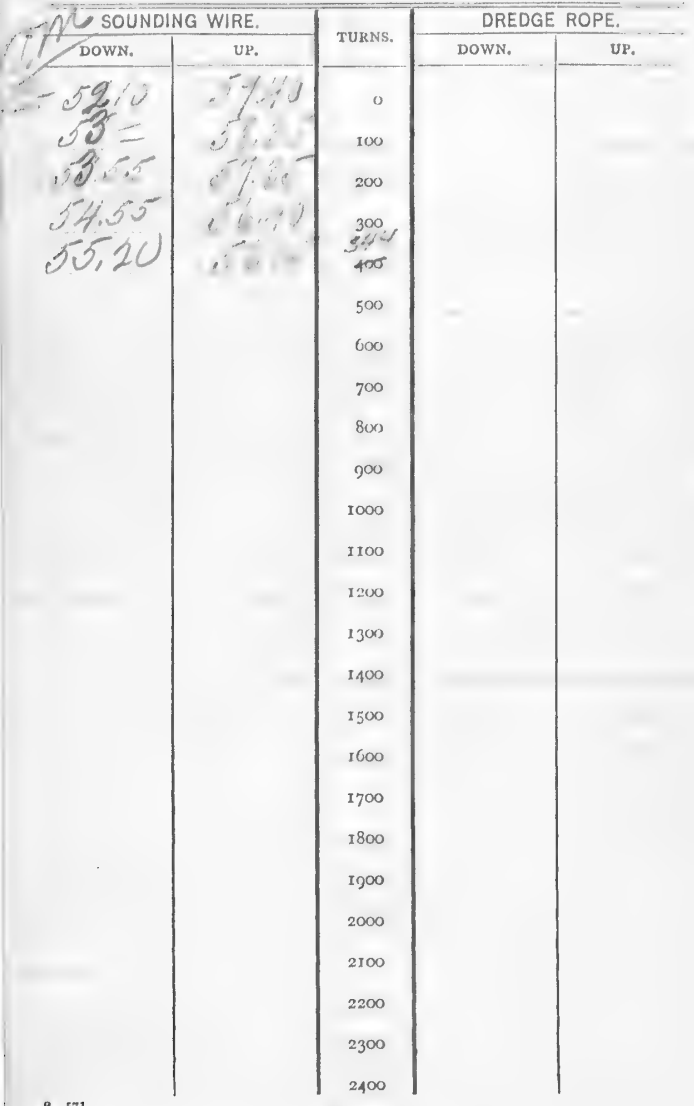


No, 0 398 Date

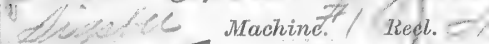
Turns $2 / 5$ cor. +221 Dopth 2,7

shoot or lead

Botitom

Bottom temperalure

No. of thormometer

Cor.

Corrected temperature

Air

817

Sicrface
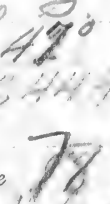

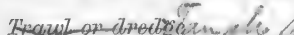
in Dift os

Sogein Drift $\mid>1 ;$

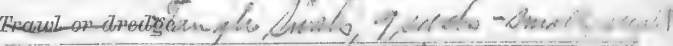

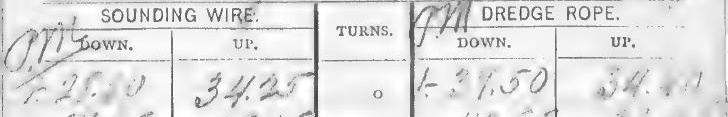

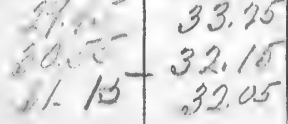

100

200

215

$7: 1$.

$\hat{7}=$

d) 1,10

400

54.45

500

58.20

600

700

800

900

1000

1100

1200

1300

1400

1500

1600

1700

I 800

IgOo

2000

2100

2200

2300

2400 
Namlewile Lify, $830^{\circ} 30^{\prime} \gamma, 3_{1}^{5} / 10^{\prime}$

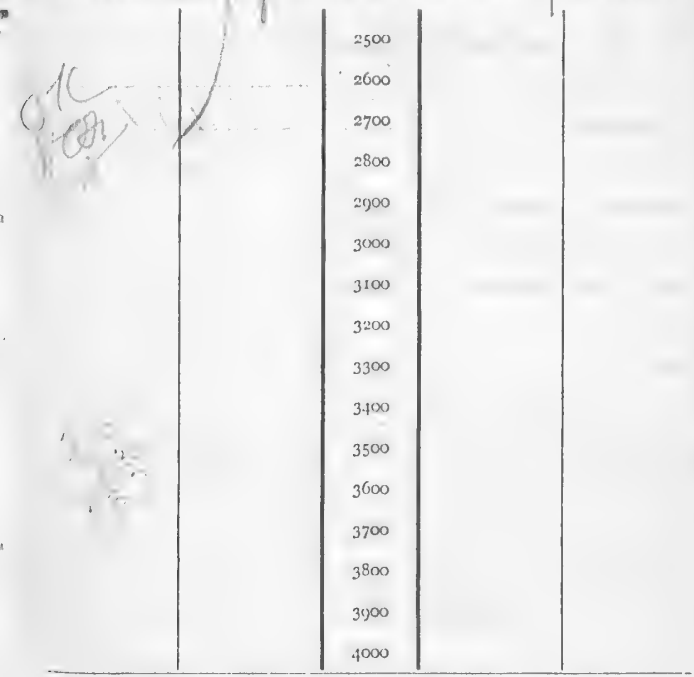

SERIAL TEMPERATURES.

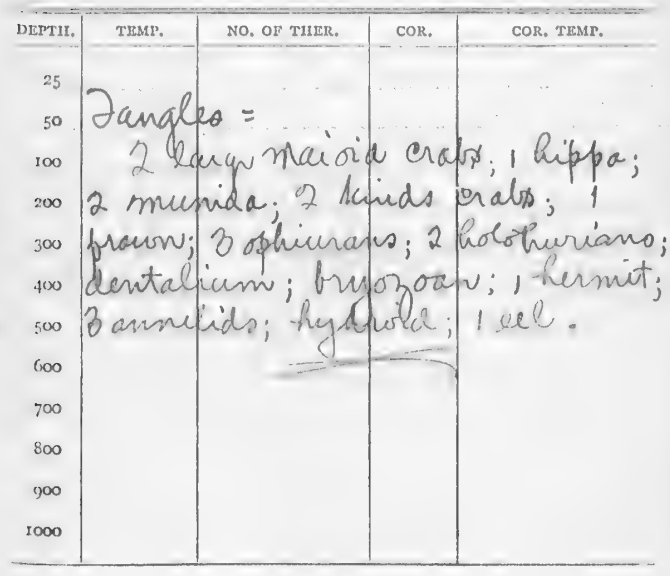

REMARKS : 
507 .

No

4427 Dato

Q Vi, : Machine. Kecl.

Turns/ 3 Cor. +

Depth

Sthob top lead

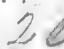

,

Bottom

Bottom temperatuleet -

No. of thermometer

Cor.

Corrected temperature

Air \& Surface

Drift

Trawl or dredge

11 SOUNDING WIRE.

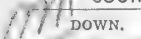

UP.

IO0

200

300

400

500

600

700

800

900

1000

IIOO

1200

1300

I 400

I 500

1600

1700

1800

1900

2000

2100

2200

2300

2400
DREDGE ROPE. DOWN.

UP. 


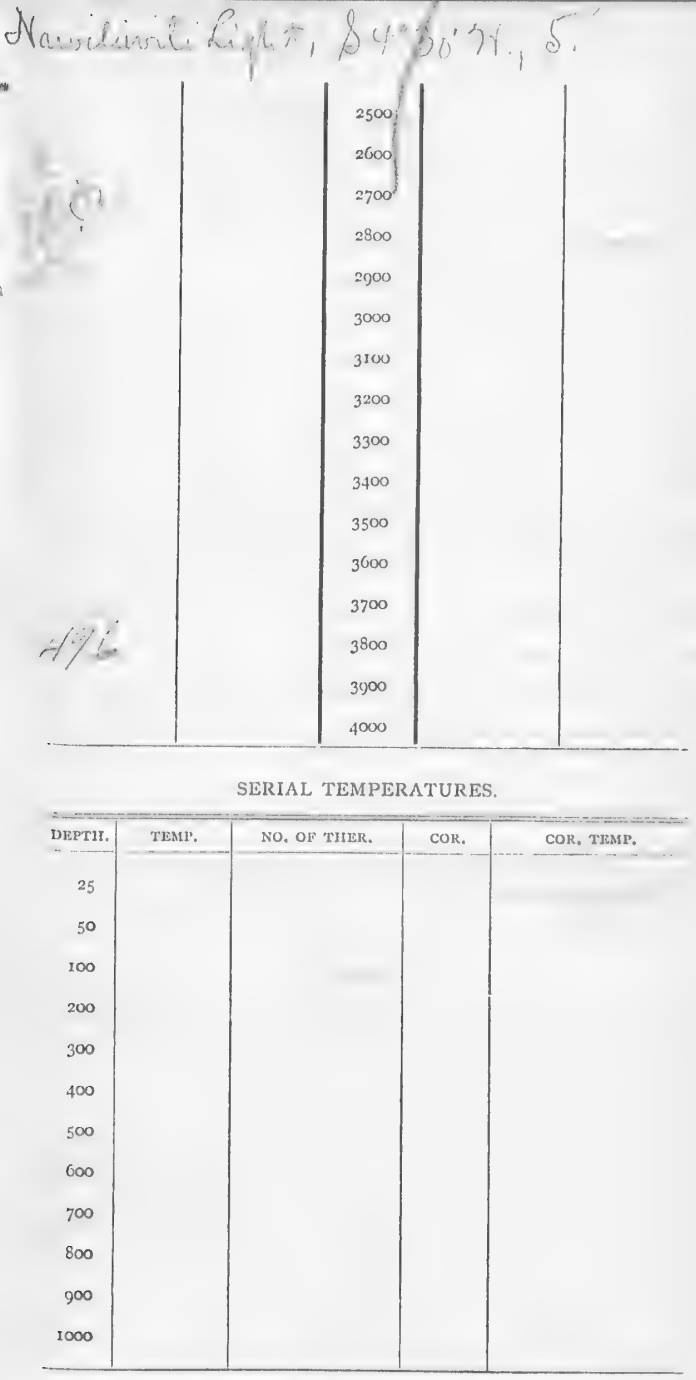

SERIAL TEMPERATURES.

REMARKS : 
Hanamaulu (thukini)'Trrehouse
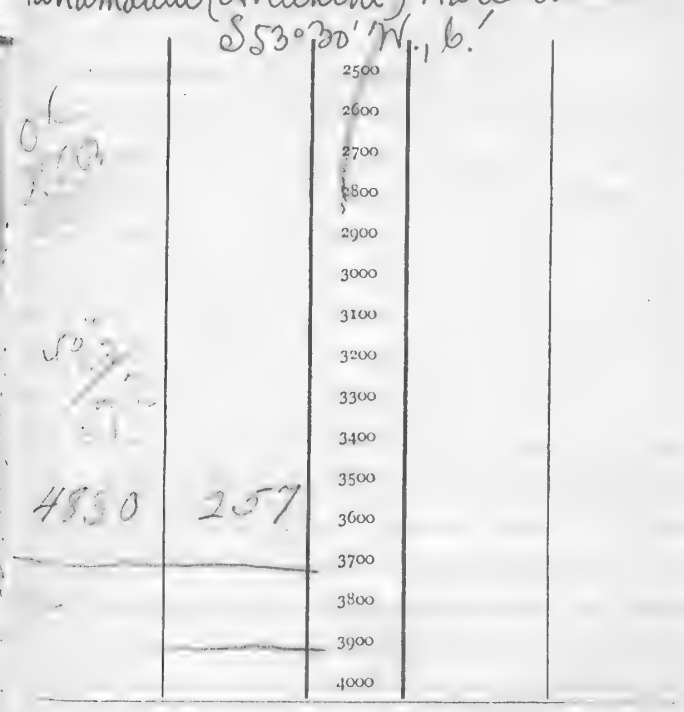

SERIAL TEMPERATURES.

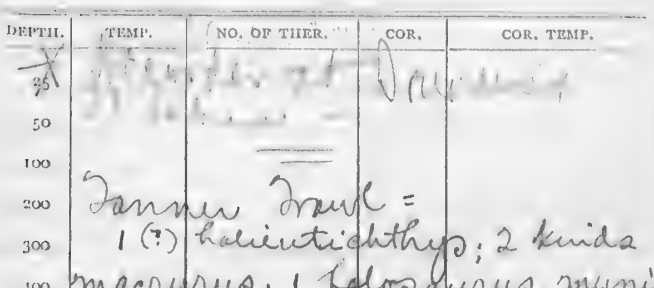
macrepruo, 1 halos curus; rncunida, 2 knids prauno; 1 folothurian;

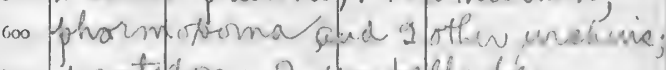
200 intifion; 2 Amtelelula.

800

900

REMARKS :

9600

Plent deritere. 
som

$1 ?$

i) $\mathrm{No}$ $33 y$

Date

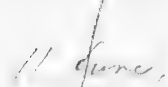
Q.?. Sirsbee Machine. K/ Reel. T, . . .

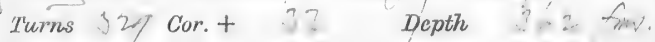
Shot or tead $35<6$.

Bottom

$7 \%+\therefore$,

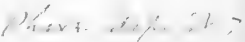

Bottom temperature

\section{8}

No. of thermometer

Corrected temperature

Air 78 Surface

Trawt on dractge $8 \%$

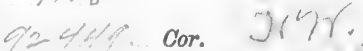

SOUNDING WIRE.

DOWN. $\frac{\text { UP. }}{7-7}$

S.

$\therefore$ is $8 . \cdots$

$\therefore<\infty$

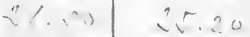

,

8

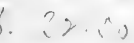

(1) $24.2 \%$

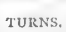

TURNS.

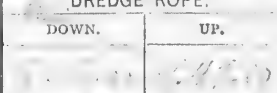

200

300

I2?

400

$5 n 0$

(.

6.

700

800

900

1000

1100

1200

1300

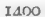

I500

1600

I 700

r 800

1900

2000

2100

2200

2300

2400 (1) $\because \because 510$ $\therefore, ?, 7$

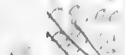
$\because$.

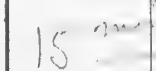
10.

\section{Drift}

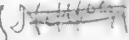

1,

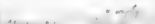

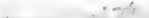




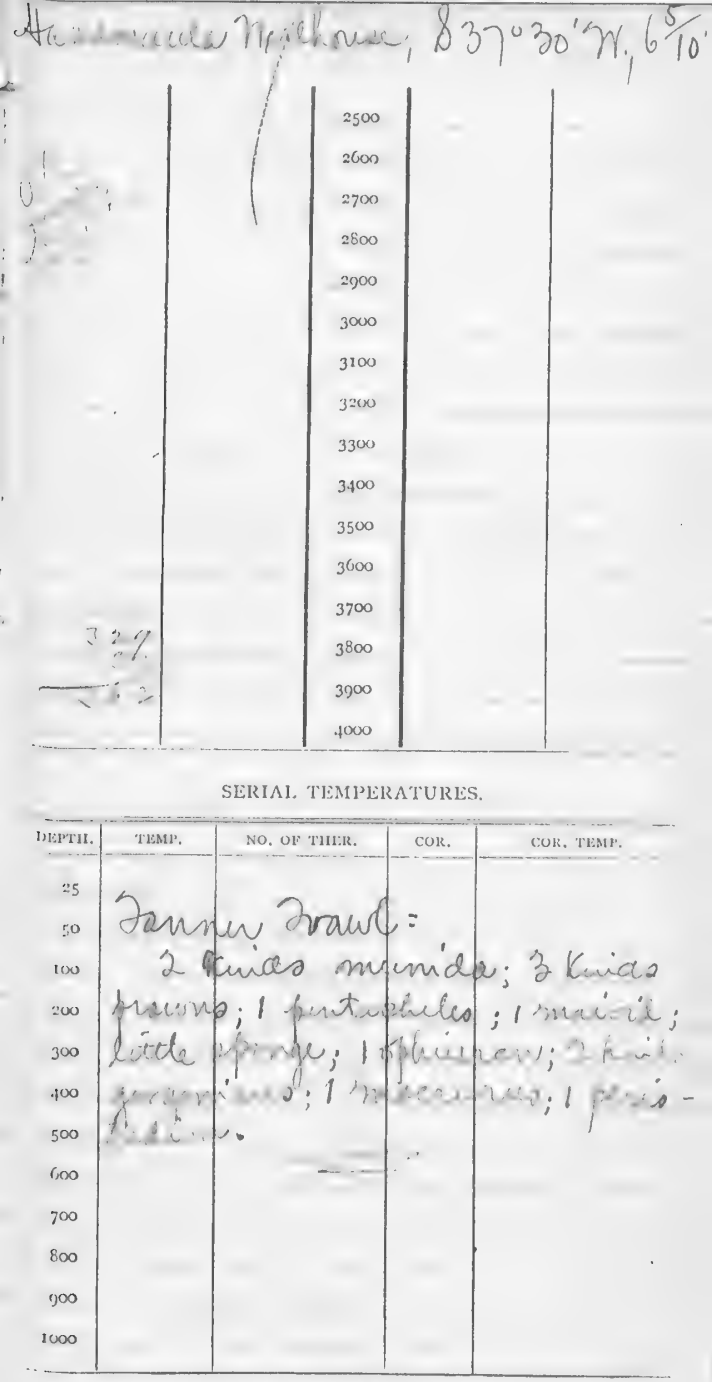

SERIAI. TEMPLRATURES

REMARKS : 
Reel.

C.si Machine.

Depth s.5 fiew.

Turns so Cor.t

strot or lead 36

Bottom , cry $c_{3} S$.

Botiom temperature.

No. of thermometer

Corrected temperature

Air . 78. Surface .7. Drift

$92 \times 49$. Cor.

$x \times 21^{n}$,

trawthor dredise

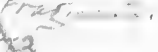

SOUNDING WIRE.

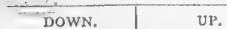

UP.

$\because, 152$

$2 C_{2}=$
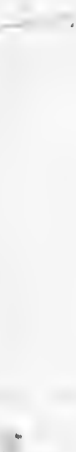
$1 / 1^{11}$

No.

Dates

0.2

1 Finer Machine. I Reel.

Turns 48 Cor.t $2 /$ Depth v's frow.

Shobor lead 26 ofo.

Bottom

cos. $\mathrm{SL}$

Bottom temperature

No. of thermometer

Lone Cor.

Corrected temperature

Air $7 \%$ Surface $\% \%$ Drift

Trawl or dredge

$\therefore$ SOUNDING WIRE.

DOWN. UP. TURNS.

1021.30

$+$
0 $\cdots$

(2)

PQ⿻

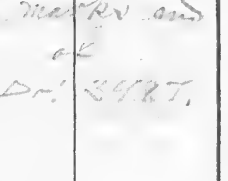

30(

400

500

600

700

800

900

1000

1100

1200

1300

$I 400$

1500

1600

1700

I 800

1900

2000

2100

2200

2300

$240 n$
DREDGE ROPE. DOWN. 
Henamaula Warchous, is $23^{\circ} \mathrm{W} .77^{5} / 10^{\prime}$

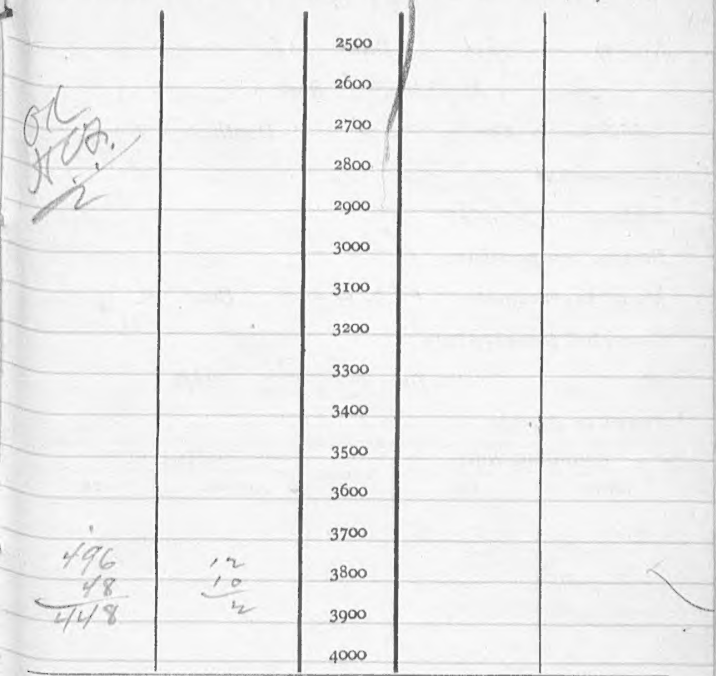

SERIAL TEMPERATURES.

\begin{tabular}{r|c|c|c|c}
\hline DEPTH. & TEMP. & NO, OF THER. & COR. & COR, TEMP. \\
\hline 25 & & & & \\
50 & & & & \\
100 & & & & \\
200 & & & & \\
300 & & & & \\
400 & & & & \\
500 & & & & \\
600 & & & & \\
700 & & & & \\
800 & & & & \\
900 & & & & \\
\hline 000 & & & & \\
\hline
\end{tabular}

REMARKS : 
Stanamaulu Warehone, $843^{\circ} 2,14 \frac{9}{10}$
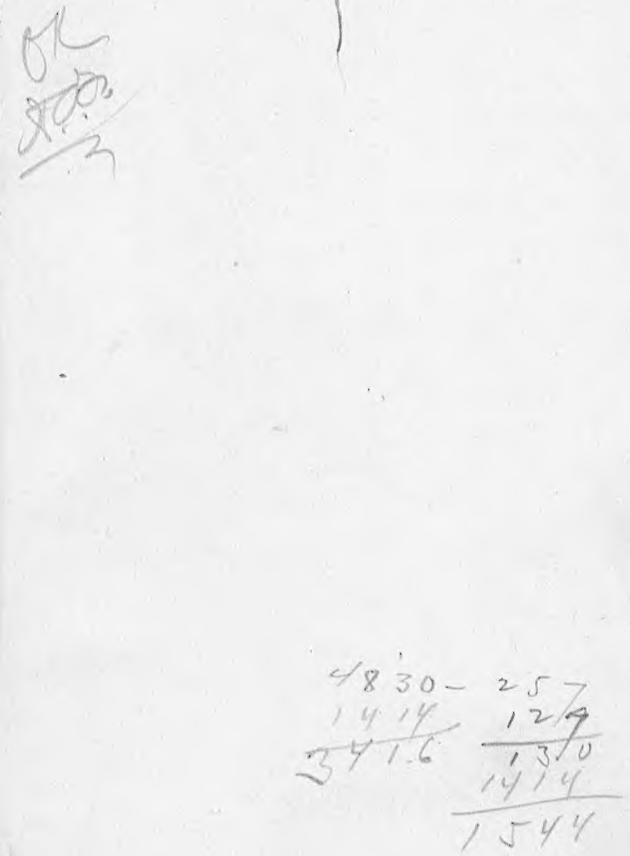


$$
\begin{aligned}
& 15-35 \\
& 5-60 \\
& \hline
\end{aligned}
$$

$$
\begin{aligned}
& 94-35 \\
& 56-60
\end{aligned}
$$

